\title{
Structural insights into recognition of chemokine receptors by Staphylococcus aureus leukotoxins
}

Paul Lambey ${ }^{1}$, Omolade Otun ${ }^{1}$, Xiaojing Cong ${ }^{1}$, François Hoh ${ }^{3}$, Luc Brunel ${ }^{3}$, Pascal Verdié ${ }^{3}$, Claire Grison ${ }^{1}$, Fanny Peysson ${ }^{1}$, Sylvain Jeannot ${ }^{1}$, Thierry Durroux ${ }^{1}$, Cherine Bechara ${ }^{1,4}$, Sébastien Granier $^{1 *}$ and Cédric Leyrat ${ }^{1 *}$

\section{${ }^{1}$ IGF, University of Montpellier, CNRS, INSERM, Montpellier, France}

${ }^{2}$ Centre de Biochimie Structurale, CNRS UMR 5048-INSERM 1054- University of Montpellier, 29 rue de Navacelles, 34090 Montpellier Cedex, France.

${ }^{3}$ Institut des Biomolécules Max Mousseron (IBMM), University of Montpellier, CNRS, ENSCM, Montpellier, France

${ }^{4}$ Institut Universitaire de France (IUF)

* Correspondence: sebastien.granier@igf.cnrs.fr and cedric.leyrat@igf.cnrs.fr

\section{Abstract}

Staphylococcus aureus (SA) leukocidin LukED belongs to a family of bicomponent pore forming toxins that play important roles in SA immune evasion and nutrient acquisition. LukED targets specific $G$ protein-coupled chemokine receptors to lyse human erythrocytes and leukocytes. The first recognition step of receptors is critical for specific cell targeting and lysis. The structural and molecular bases for this mechanism are not well understood but could constitute essential information to guide antibiotic development. Here, we characterized the interaction of LukE with chemokine receptors ACKR1, CCR2 and CCR5 using a combination of structural, pharmacological and computational approaches. First, crystal structures of LukE in complex with a small molecule mimicking sulfotyrosine side chain ( $p$-cresyl sulfate) and with peptides containing sulfotyrosines issued from receptor sequences revealed the location of receptor sulfotyrosine binding sites in the toxins. Then, by combining the available experimental information with protein docking, classical and accelerated weight histogram (AWH) molecular dynamics we propose models of the ACKR1-LukE and CCR5-LukE complexes. This work provides novel insights into chemokine receptor recognition by leukotoxins and suggests that the conserved sulfotyrosine binding pocket could be a target of choice for future drug development. 


\section{Introduction}

Staphylococcus aureus (SA) is a major opportunistic human pathogen that causes a wide range of clinical manifestations (Tong, Davis et al. 2015), and poses growing health concern due to the emergence of multidrug resistant strains. Pathogenic SA strains produce a number of wallassociated or secreted virulence factors that promote growth, nutrient acquisition and evasion of the host immune system (DeLeo, Diep et al. 2009, Oliveira, Borges et al. 2018). Among this arsenal of virulence factors, the bicomponent leukotoxins are $\beta$-barrel pore forming toxins that play an important role in SA pathogenesis by targeting host leukocytes and erythrocytes (Spaan, van Strijp et al. 2017), and appear to be promising targets for drug development (Kong, Neoh et al. 2016). Human clinical SA isolates can produce up to five different leukotoxins: $y$-hemolysin $A B$ and $C B$ ( $H I g A B$ and HIgCB), Leukocidin ED (LukED), Panton-Valentine Leukocidin (PVL or LukSF-PV), and Leukocidin AB (LukAB, also known as LukGH). Each leukotoxin is made up of two subunits of approximately $30 \mathrm{kDa}$ : a host cell targeting S- component (HIgA, HIgC, LukE, lukS-PV and LukA/G), and a polymerization Fcomponent (HIgB, LukD, lukF-PV and LukB/H), which can bind the plasma membrane through highly conserved phosphocholine binding sites (Olson, Nariya et al. 1999, Liu, Kozhaya et al. 2020). S- and F-components typically display relatively high conservation within each group ( $70 \%$ sequence identity), but sequence identity drops below $30 \%$ between groups (Spaan, van Strijp et al. 2017).

With the exception of LukGH, these toxins are secreted as monomers that bind target cells and subsequently octamerize into a lytic pore on the plasma membrane, composed of alternating S- and F- subunits (Olson, Nariya et al. 1999, Guillet, Roblin et al. 2004, Yamashita, Kawai et al. 2011, Yamashita, Sugawara et al. 2014, Nocadello, Minasov et al. 2016, Trstenjak, Milic et al. 2020). Each toxin is composed of 2 domains: a central $\beta$ sandwich called the CAP domain and involved in inter-protomer interactions, and a RIM domain which contains several divergent loops driving host cell specificity. The STEM region is a subdomain of the CAP, which changes its conformation upon pore formation to associate with neighboring protomers and form a $\beta$ barrel that inserts into the cell membrane (Yamashita, Kawai et al. 2011, Yamashita, Sugawara et al. 2014).

Over the last decade, proteinaceous receptors have been identified for most of the bicomponent leukotoxins, providing key insights into their role in SA pathogenesis and the mechanisms driving their cellular tropism and species specificity (Spaan, van Strijp et al. 2017). Specifically, HIgA and LukE were shown to share atypical chemokine receptor 1 (ACKR1) as their main receptor on erythrocytes (Spaan, Reyes-Robles et al. 2015), while recruiting CCR5 (LukE), CCR2 (HIgA), CXCR1 and CXCR2 (both HIgA and LukE) to differentially target specific leukocyte populations (Alonzo, Kozhaya et al. 2013, Reyes-Robles, Alonzo et al. 2013, Spaan, Vrieling et al. 2014). Conversely, HIgC and LukS-PV were found to bind monocytes and neutrophils through C5aR1 and C5aR2 (Spaan, Henry et al. 2013, Spaan, Vrieling et al. 2014). All of the identified receptors belong to the chemokine or complement receptor families of class A G-protein-coupled receptors (GPCRs), except for the divergent lukGH toxin which binds CD11b (aka complement receptor 3) expressed on phagocytic cells (DuMont, Yoong et al. 2013). Recently, F-component-specific receptors have been identified as well: human CD45 was shown to act as a receptor for lukF-PV (Tromp, Van Gent et al. 2018), while HlgB was found to interact with ACKR1 (Grison, Leyrat et al. 2021).

Several of these toxin-GPCR pairs have been characterized at the molecular level by swapping mutagenesis of divergent regions/loops (DRs) of the rim domain, in particular for LukE and 
HIgA that can target multiple chemokine receptors (Reyes-Robles, Alonzo et al. 2013, Laventie, Guerin et al. 2014, Tam, Schultz et al. 2016, Peng, Takeshita et al. 2018, Vasquez, Lubkin et al. 2020). These studies highlighted the critical role of several DRs located in the rim domain in determining receptor specificity. From the receptors side, residues of CCR5 were identified in eCL2 and in the upper part of TM7 that lead to severe loss of LukED activity (Tam, Schultz et al. 2016). Several residues of ACKR1 were also identified by mutagenesis, the most critical being the sulphated tyrosine sTyr41 for which the alanine substitution nearly abolished LukED and HIgAB activity on erythrocytes (Spaan, Reyes-Robles et al. 2015). Interestingly, all of the GPCRs that bind leukotoxins possess between 1 and 4 potentially sulfated tyrosine residues in their N-terminal regions, and the interaction between LukS-PV and the C5aR1 N-terminal region was shown to require tyrosine sulfation (Spaan, Henry et al. 2013). In addition, posttranslational modification pathways involved in the sulfation of the leukotoxin receptors were recently found to impact on HIgAB, HIgCB, LukED, and PVL induced cytotoxicity (Tromp, Van Gent et al. 2020). Despite these advances, a structural understanding of toxin-chemokine receptors interactions is still lacking due to the unavailability of high resolution structures of the complexes.

In the present study, we provide a detailed characterization of the structure and dynamics of monomeric LukE using integrated structural biology methods. We show that recombinant LukE is able to displace bound chemokines from ACKR1, CCR5 and CCR2 on the cell surface using a TR-FRET based approach. We next solved the crystal structures of LukE in complex with a small molecule mimicking a sulfotyrosine side chain ( $p$-cresyl sulfate) and with sulfopeptides derived from the $\mathrm{N}$-terminal region of ACKR1 and CCR2, revealing two conserved sulfotyrosine binding sites. Finally, we use computational docking and molecular dynamics (MD) simulations to propose models of the ACKR1-LukE and CCR5-LukE complexes that are consistent with available experimental data.

\section{Results}

\section{Solution and crystallographic structures of LukE}

The crystal structure of monomeric LukE has previously been reported at $3.2 \AA$ resolution (PDB code 3ROH) (Nocadello, Minasov et al. 2016). While attempting to co-crystallize LukE in complex with peptides encompassing the sulfated ACKR1 Tyr41 residue, we serendipitously obtained two additional apo crystal forms of LukE at $1.5 \AA$ (apo1) and $1.9 \AA$ (apo2), allowing us to build a higher quality model (see table 1 and methods). The apo2 structure, like the original $3 \mathrm{ROH}$ structure, belongs to space group 14 , while the apo1 structure crystallized in $\mathrm{P} 2{ }_{1} 2_{1} 2_{1}$ and features an entirely different crystal packing (figure $\mathbf{1 A}$ and figure S1). All three structures contain one copy of the protein in the asymmetric unit and display very similar conformations, with $\mathrm{C} \alpha \mathrm{RMSDs}$ of $1.18 \AA$ (apo1) and $0.81 \AA$ (apo2) relative to the $3 \mathrm{ROH}$ structure (figure 1A). One notable difference is the presence of residues $12-23$ belonging to the N-terminal signal sequence in apo2, which are involved in crystal packing interactions, possibly accounting for the higher resolution compared to the $3 \mathrm{ROH}$ structure (figure $1 \mathrm{~A}$ and figure S1). A comparison of the B-factors of the three structures is shown in figure S1DEF, highlighting the influence of crystal packing on protein flexibility within the crystals.

In order to obtain structural information about LukE directly in solution, we used small angle $\mathbf{x}$-ray scattering (SAXS). The experimental SAXS profiles are shown in figure 1B. The samples 
were free from aggregation as evidenced by the linearity of the Guinier region, however the measured radius of gyration $(\mathrm{Rg})$ increased from $2.41 \pm 0.02 \mathrm{~nm}$ at $1 \mathrm{mg} / \mathrm{ml}$ to $2.68+/-0.08$ at $4 \mathrm{mg} / \mathrm{ml}$ of protein, suggesting a slight tendency for interparticle attraction. This was consistent with a native mass spectrum of LukE showing a small proportion of dimeric forms in equilibrium with LukE monomers (figure S2). In order to extract structural information from the SAXS data we turned to the ensemble optimization method (EOM) using ensembles of models derived from atomistic MD simulations (figure $1 C D$, and figure S1 GH I). The MD generated models of LukE were fitted to a merged SAXS curve to remove the interparticle attraction effects (figure 1B), yielding a $\chi$ value of 0.79 . Rg values of the selected models were in a narrow range between 2.40 and $2.55 \mathrm{~nm}$ (Figure 1D), consistent with the values of 2.32 and $2.43 \mathrm{~nm}$ calculated from the apo1 and apo 2 structures. Conformations of the models from the optimized ensemble show a very rigid core, with highly flexible terminal regions corresponding to the signal peptide and polyhistidine tag. Localized flexibility is also present in the divergent loops of the RIM domain, and in the STEM region loop (residues 151-159) (figure 1B and figure S1). The three $\beta$ strands of the STEM remain however relatively rigid, suggesting that specific conformational triggers are required to explore the extended conformations observed in leukotoxin pore structures.

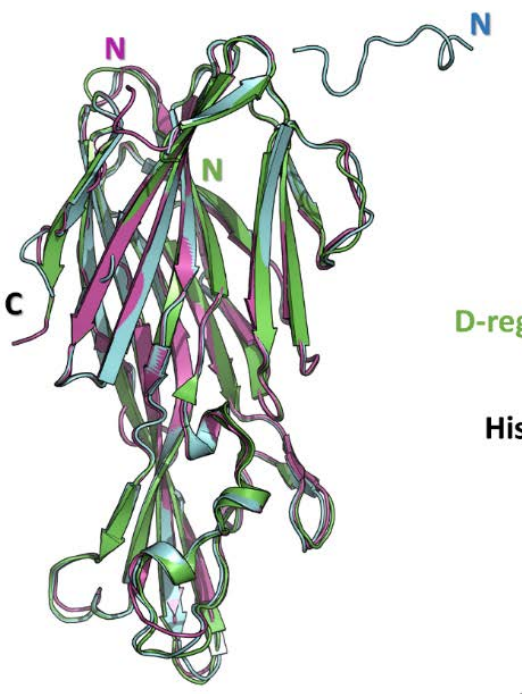

C

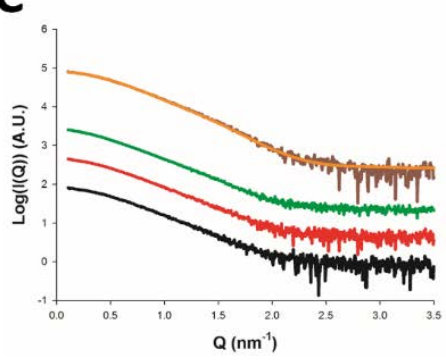

B
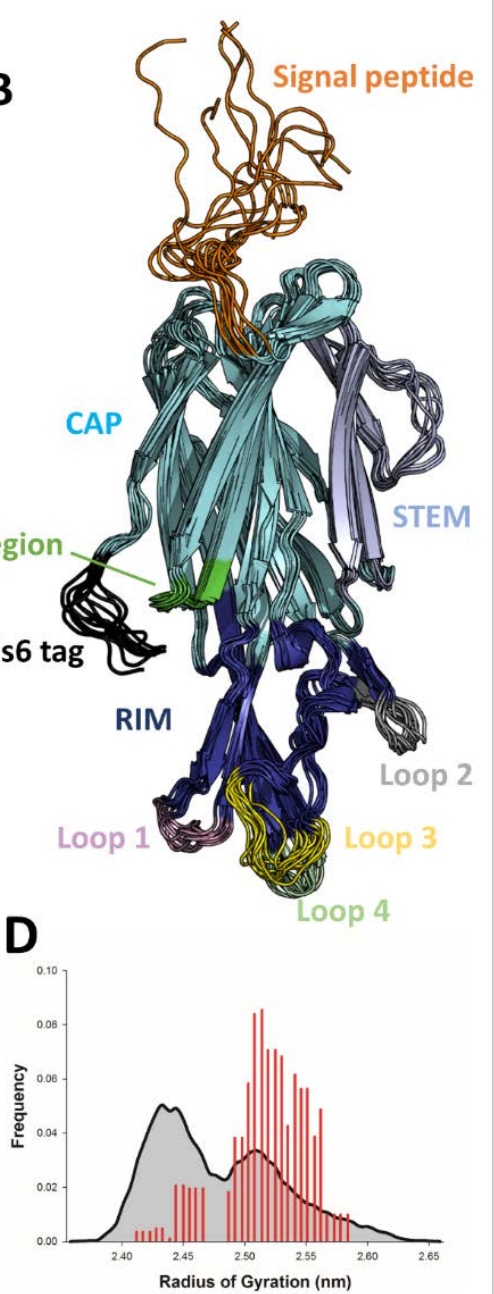

Figure 1: Solution and crystallographic structures of LukE. A. The crystal structures of LukE in P $2{ }_{1} 2_{1} 2_{1}$ (Apo 1, green) and 14 (Apo 2, cyan) space groups are shown in cartoon representation and overlaid onto the previously published crystal structure of LukE (magenta), also in 14 space group (PDB code $3 R O H$ ). B. Optimized ensemble 
of 10 models corresponding to the fitted SAXS profile in C. The N-terminal extension belonging to the signal peptide and the C-terminal polyhistidine tag are shown in orange and black, respectively. The CAP, RIM and STEM domains are coloured in cyan, deep blue and light blue, respectively. Other regions of interest are also indicated such as the D-region, and divergent loops 1-4. C. Small angle x-ray scattering (SAXS) profiles of LukE measured at 1,2 and $4 \mathrm{mg} / \mathrm{mL}$ are shown as black, red and green lines, respectively. The merged SAXS curve is shown as a brown line with the fitting curve obtained using the ensemble optimization method (EOM) in orange. D. Radius of gyration distributions for the initial pool ensemble (gray area and black line) and for the optimized ensembles (red bars) obtained using the merged SAXS curve.

\begin{tabular}{|c|c|c|c|c|c|}
\hline & Apo1 & Apo2 & $\begin{array}{l}\text { p-cresol sulfate } \\
\text { soak }\end{array}$ & $\begin{array}{l}\text { AcDsYDsYG-NH2 } \\
\text { soak }\end{array}$ & $\begin{array}{l}\text { AcDSFPDGDsY } \\
\text { GANLE-NH2 soak }\end{array}$ \\
\hline \multicolumn{6}{|l|}{ Data collection } \\
\hline Space group & $\mathrm{P} 22_{1} 2_{1} 2_{1}$ & 14 & $\mathrm{P} 22_{1} 2_{1} 2_{1}$ & $\mathrm{P} 2{ }_{1} 22_{1}$ & $\mathrm{P} 21212_{1}$ \\
\hline \multicolumn{6}{|l|}{$\begin{array}{l}\text { Cell } \\
\text { dimensions }\end{array}$} \\
\hline$a, b, c(\AA ̊)$ & $62.58,72.20,78.81$ & $\begin{array}{l}135.79,135.79 \\
63.53\end{array}$ & $62.58,73.49,79.34$ & $63.30,72.41,79.00$ & $62.97,71.18,79.23$ \\
\hline$\alpha, \beta, \gamma\left({ }^{\circ}\right)$ & $90.00,90.00,90.00$ & $90.00,90.00,90.00$ & $90.00,90.00,90.00$ & $90.00,90.00,90.00$ & $90.00,90.00,90.00$ \\
\hline Resolution $(\AA ̊)$ & $\begin{array}{l}49.01-1.46(1.48- \\
1.46)\end{array}$ & $\begin{array}{l}48.01-1.90(1.94- \\
1.90)\end{array}$ & $\begin{array}{l}47.65-1.60(1.63- \\
1.60)\end{array}$ & $\begin{array}{l}49.40-1.40(1.42- \\
1.40)\end{array}$ & $47.16-1.55(1.58-1.55)$ \\
\hline$R_{\text {merge }}$ & $0.079(2.601)^{\mathrm{NA}}$ & $0.100(1.510)^{\mathrm{NA}}$ & $0.040(0.710)$ & $0.038(1.129)^{\mathrm{NA}}$ & $0.032(0.811)$ \\
\hline $\mathrm{CC}_{1 / 2}$ & $1.000(0.508)$ & $0.984(0.748)$ & $0.996(0.624)$ & $0.999(0.483)$ & $0.998(0.622)$ \\
\hline$l / \sigma l$ & $17.2(1.0)$ & $19.2(1.9)$ & $15.5(1.7)$ & $15.8(1.2)$ & $17.5(1.5)$ \\
\hline $\begin{array}{l}\text { Completeness } \\
(\%)\end{array}$ & $99.9(98.5)$ & $100(100)$ & $99.3(99.2)$ & $99.2(97.3)$ & $99.2(99.8)$ \\
\hline Redundancy & $13.1(12.0)$ & $13.7(13.8)$ & $3.4(3.1)$ & $4.1(3.6)$ & $3.2(3.3)$ \\
\hline \multicolumn{6}{|l|}{ Refinement } \\
\hline Resolution (Å) & 49.1-1.46 & $48.1-1.90$ & $47.7-1.60$ & $40.9-1.40$ & $47.2-1.55$ \\
\hline No. reflections & 62775 & 45734 & 48967 & 72136 & 52377 \\
\hline$R_{\text {work }} / R_{\text {free }}$ & $17.81 / 19.41$ & $18.63 / 20.30$ & $18.12 / 20.46$ & $18.44 / 19.81$ & $18.12 / 20.37$ \\
\hline \multicolumn{6}{|l|}{ No. atoms } \\
\hline \multirow[t]{2}{*}{ Protein } & 2370 & 2472 & 2351 & 2359 & 2276 \\
\hline & 0 & 0 & 57 & 71 & 140 \\
\hline \multicolumn{6}{|l|}{ Ligand/peptide } \\
\hline Water & 328 & 323 & 260 & 293 & 215 \\
\hline \multicolumn{6}{|l|}{$B$-factors } \\
\hline \multirow[t]{2}{*}{ Protein } & 28.3 & 37.5 & 32.8 & 31.7 & 36.9 \\
\hline & NA & NA & 29.6 & 31.8 & 38.3 \\
\hline \multicolumn{6}{|l|}{ Ligand/peptide } \\
\hline Water & 44.3 & 53.3 & 48.4 & 47.8 & 51.1 \\
\hline \multicolumn{6}{|l|}{$\begin{array}{l}\text { R.m.s. } \\
\text { deviations }\end{array}$} \\
\hline $\begin{array}{l}\text { Bond lengths } \\
\text { (Å) }\end{array}$ & 0.009 & 0.009 & 0.010 & 0.010 & 0.010 \\
\hline Bond angles & 1.108 & 1.092 & 1.102 & 1.226 & 1.163 \\
\hline
\end{tabular}




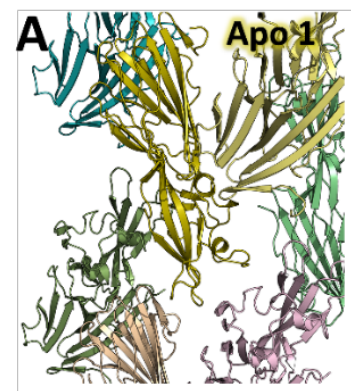

D

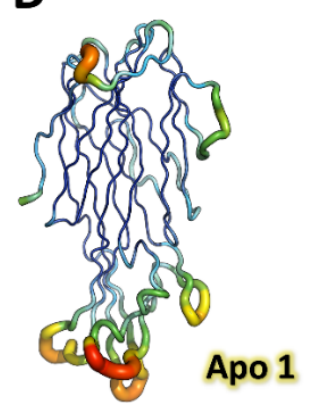

G

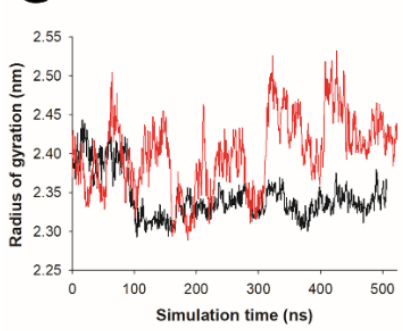

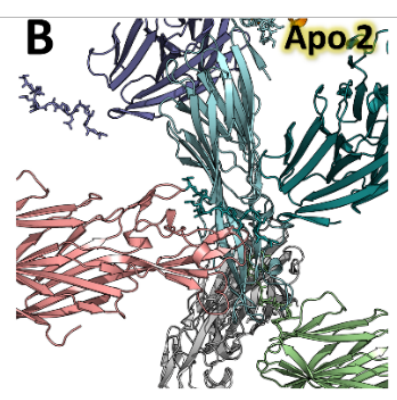

E

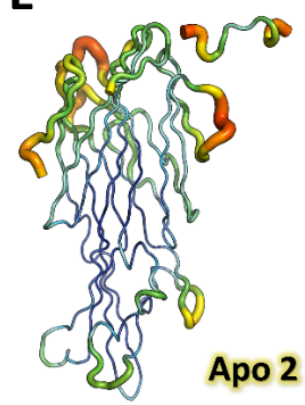

Apo 2

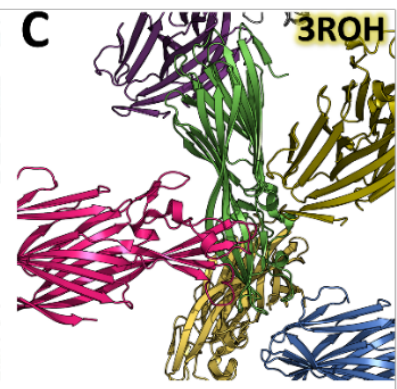

$\mathbf{F}$

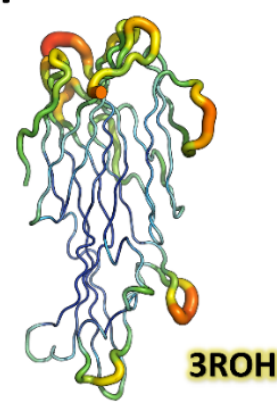

3ROH
$\mathbf{H}$

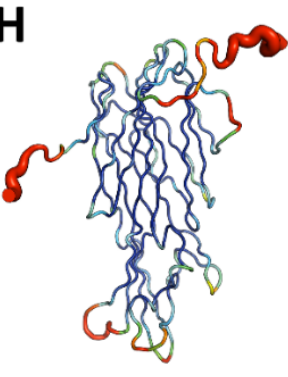

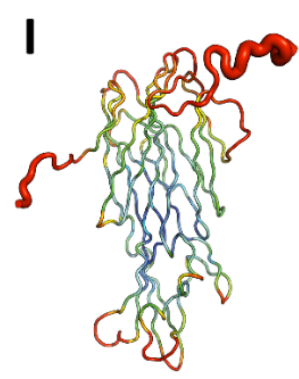

Supplementary figure 1: Crystallographic packing differences between LukE structures, LukE flexibility in crystal structures and molecular dynamics simulations. A, B and C. Crystallographic packing differences between LukE apo 1 structure in $P 2{ }_{1} 2{ }_{1}{ }_{1}$ space group $(A)$, apo $2(B)$ and the previously published structure (C) (PDB code: $3 \mathrm{ROH})$, both in 14 space group. D, E and F. B-factor putty representation of the apo 1, apo 2 and 3ROH crystal structures, highlighting the flexibility of the stem loop and the DR loops within the rim domain, as well as the influence of crystal packing on the flexible regions. G, H and I. Molecular dynamics simulations data of LukE. (G) Radius of gyration ( $\mathrm{Rg}$ ) over time is shown for two independent MD trajectories performed with the amber99sbildn (black line) or amber99sbw (red line). The amber99sbw force field was parameterized with rescaled waterprotein interactions to improve the sampling of intrinsically disordered regions (reference), avoiding the collapse of LukE N-terminal signal peptide and C-terminal polyhistidine tag onto the protein core. $\mathrm{H}$ and I. B-factor putty representation of the root mean square fluctuations of LukE extracted from the amber99sb-ildn $(\mathrm{H})$ or amber99sbw (I) trajectories. 


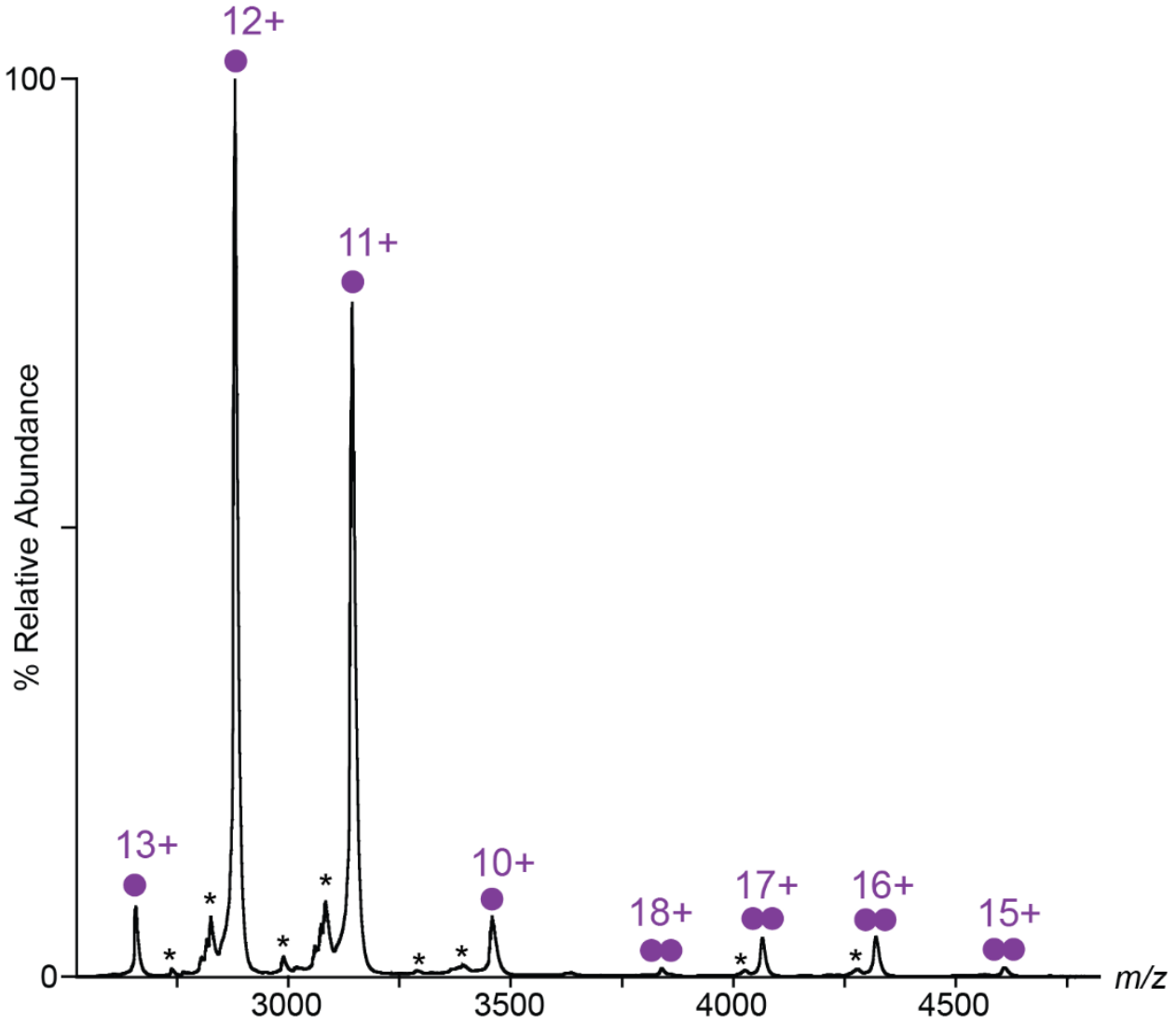

Supplementary figure 2: Native MS spectrum of LukE. nMS spectrum of $20 \mu \mathrm{M}$ LukE showing the presence of both monomeric species (single purple circle) at $34508 \pm 4$ Da and dimeric species (double purple circle) at $69108 \pm 8 \mathrm{Da}$. Theoretical mass of LukE is $34506 \mathrm{Da}$. hydrolysed isoforms of lukE are also visible at low intensities (asterisks).

LUkE competes with CCL5 binding onto ACKR1 and CCR5, but also with CCL2 binding onto CCR2

We next sought to analyze the binding of LukE to its human chemokine receptor targets. A wealth of experimental information is available indicating that the expression of CCR5, ACKR1, CXCR1 and CXCR2 render human cells susceptible to LukED killing (Alonzo, Kozhaya et al. 2013, Reyes-Robles, Alonzo et al. 2013, Spaan, Reyes-Robles et al. 2015, Tam, Schultz et al. 2016, Vasquez, Lubkin et al. 2020). Direct interaction between LukE and CCR5 or ACKR1 has also previously been characterized by surface plasmon resonance (SPR) in purified systems, indicating Kd values around 40 and $60 \mathrm{nM}$, respectively (Alonzo, Kozhaya et al. 2013, Vasquez, Lubkin et al. 2020). In order to complement the available experimental data and to verify that our recombinantly produced LukE is biologically active, we performed competitive binding assays in HEK293T cells using Homogenous Time Resolved FRET (TR-FRET) technology (Zwier, Roux et al. 2010) (figure 2). For these experiments, we selected ACKR1 and CCR5, which are major leukotoxin receptors in erythrocytes and leukocytes, but also CCR2 which is normally an in vivo target of HIgA rather than LukE (Spaan, Vrieling et al. 2014). SNAP-tag-fused CCR5, ACKR1 and CCR2 receptors transiently expressed in HEK293 cells were covalently labelled with Lumi4-terbium as donor. Cells were then incubated in the presence of $5 \mathrm{nM}$ tracer chemokines d2-CCL5 (CCR5 and ACKR1) or d2-CCL2 (CCR2). Addition of increasing concentrations of LukE 
led to a decrease in the TR-FRET ratio for all receptors indicating the ability of LukE to bind competitively with IC50s of $231.7 \mathrm{nM} \pm 0.19,230.4 \mathrm{nM} \pm 0.14$ and $64.2 \mathrm{nM} \pm 0.14$ at CCR5, ACKR1 and CCR2 respectively. These results indicate at least partial overlap between the LukE and CCL2/5 binding sites on the receptors extracellular surfaces and are consistent with previous observations regarding ACKR1- and CCR5- LukE interactions (Alonzo, Kozhaya et al. 2013, Spaan, Reyes-Robles et al. 2015). Interestingly, LukE inhibited CCR2-CCL2 interaction interaction between CCR2 and LukE.
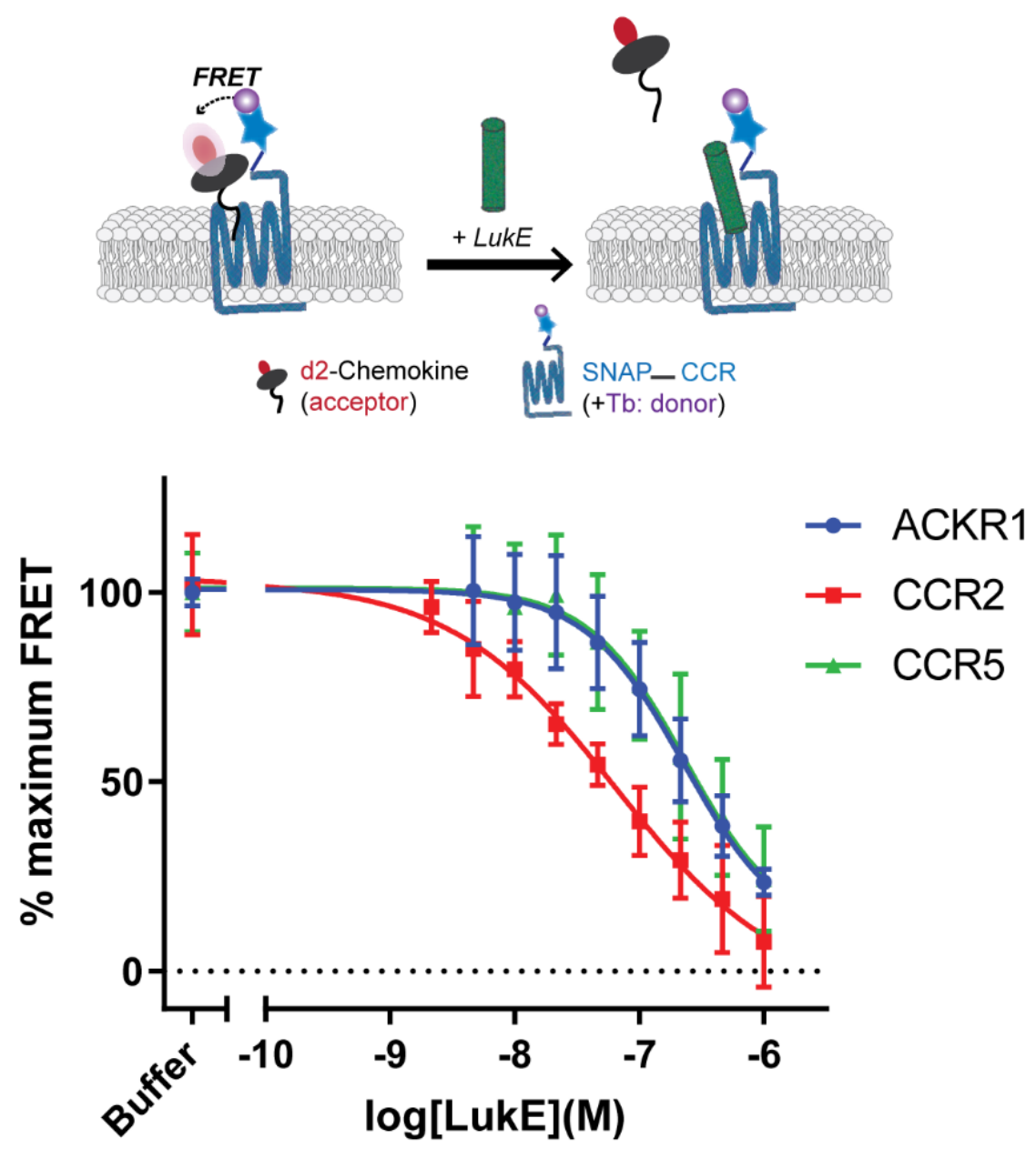

Figure 2: Binding of LukE in live cells as determined by competition TR-FRET. The upper panel shows a schematic of the competitive TR-FRET assay. Addition of toxins disrupts energy transfer between a SNAP-tagged receptor labelled with a Terbium donor and a d2-chemokine acceptor (Zwier, Roux et al. 2010). The bottom panel shows the competition dose-response curves at receptors CCR5, ACKR1 and CCR2. $5 \mathrm{nM}$ tracer ligands, CCL5-d2 for CCR5 and ACKR1 and CCL2-d2 for CCR2 were used to determine TR-FRET at their respective receptors in the presence of LukE. IC50 values are quoted in-text. Data shown is mean \pm SD of three independent experiments performed in triplicate. 


\section{Crystal structure of LukE in complex with $p$-cresyl sulfate reveals three potential sulfotyrosine binding sites}

In order to obtain structural insights into the interaction of LukE with its receptors, we carried out crystal soaking experiments using apo1 LukE crystals. Because recognition of sulfated tyrosines appears to be a common trend among leukotoxins, we selected 3 synthetic molecules for soaking, in an attempt to identify potential sulfotyrosine binding sites: (1) paracresyl sulfate (pCS) is a metabolite of tyrosine and a uremic toxin (Gryp, Vanholder et al. 2017). Its chemical structure is identical to that of a sulfotyrosine side chain ( $C \beta$ replaced by a methyl group). (2) the ${ }^{34}$ DSFPDGDsYGANLE ${ }^{46}$ peptide of ACKR1, with a functionally critical sulfated tyrosine in position $41 ;(3)$ the ${ }^{25}$ DsYDsYG ${ }^{29}$ peptide of CCR2 bearing 2 sulfated tyrosines in position 26 and 28. Although CCR2 is normally an in vivo target of HIgA rather than LukE, Tam and coworkers have shown that cells expressing WT CCR5 or a CCR5 chimera harbouring the CCR2 N-terminal region display the same susceptibility to LukED intoxication (Tam, Schultz et al. 2016). This interchangeability of the $\mathrm{N}$-terminal region is perhaps not so surprising given that both sequences contain multiple tyrosine residues that can be efficiently sulfated (Farzan, Mirzabekov et al. 1999, Preobrazhensky, Dragan et al. 2000, Tan, Ludeman et al. 2013). Crystals soaked with all 3 compounds yielded good diffraction data (table 1) that revealed strong additional electron density corresponding to the ligands (figure S3).

The structure of pCS-bound LukE is shown in figure 3. Three pCS molecules interact with LukE at distinct sites, which we named site 1, 2 and 3. Site 1 forms a surface pocket with Arg263 sidechain sitting at the bottom and engaging in a salt bridge with the pCS sulfate group (figure 3C). Arg85 and Arg290 guanidinium groups additionally flank the sulfate oxygens, and the pCS aromatic ring packs against the hydrophobic sidechains of Ile103, Leu265 and Phe287. The upper part of the pocket is delimitated by Lys52 and Trp53, which shield the pCS interacting arginines from the solvent. These residues correspond to Arg24 and Leu25 in HIgA and are part of the D-region that plays an important role in haemolytic activity (Nariya and Kamio 1997, Peng, Takeshita et al. 2018).

Site 2 is a relatively flat area located approximately $8 \AA$ below site 1 (figure 3D). The pCS sulfate is stabilized by polar contacts with Tyr269 and Arg101, and Arg101 additionally stacks its guanidinium group against the pCS phenyl ring, engaging in cation- $\pi$ interaction. Ile103 and Phe287 from site 1 are also involved in hydrophobic contacts with the pCS phenyl ring. Ser252 from a crystallographically related molecule further stabilizes pCS binding through polar contacts with the sulfate group. 


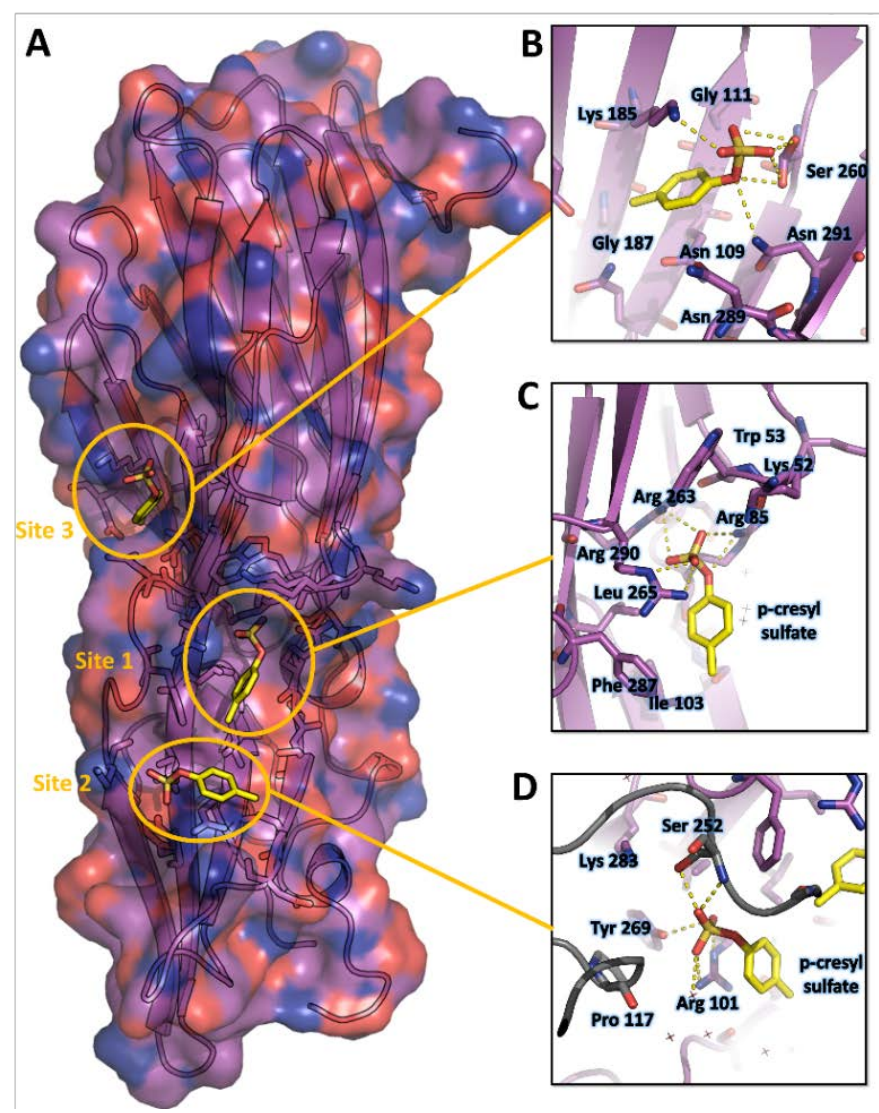

Figure 3: Crystal structure of LukE in complex with p-cresyl sulfate. A. Overview of the asymmetric unit of the crystal. Protein is shown in cartoon representation and colored magenta. The $p$-cresol sulfate molecules are shown in yellow sticks with surrounding protein residue sidechains in magenta lines $B, C$ and $D$. Close ups of the sulfotyrosine binding sites. The color code is the same as in A with p-cresol sulfate molecules and protein sidechains shown in sticks. Polar contacts between ligand and protein are shown as yellow dashed lines. In $D$, protein residues from an interacting symmetry related molecule are shown in grey cartoon and sticks.

Site 3 corresponds to a small groove in between Lys 185, Asn109, Asn291 and Ser260 sidechains (figure 3B). The pCS molecule is mainly stabilized by polar interactions of the sulfate group with with Lys 185, Asn109 and Ser260, as well as hydrophobic contact between the phenyl ring and the aliphatic part of Lys185 sidechain.

\section{Crystal structures of LukE in complex with the sulfated peptides ${ }^{34}$ DSFPDGDSYGANLE ${ }^{46}$ of ACKR1 and ${ }^{25}$ DsYDsYG ${ }^{29}$ of CCR2 show sulfotyrosine binding at site 1 and 2}

The structure of LukE in complex with the ACKR1 peptide ${ }^{34}$ DSFPDGDsYGANLE ${ }^{46}$ is shown in figure 4A. Unexpectedly, we found 2 bound copies of the peptide, which may be due to the potentially very high peptide concentration used during crystal soaking experiments (see methods). Residues 34 to 43 , and residues 39 to 46 are visible for the $1^{\text {st }}$ and $2^{\text {nd }}$ peptide, respectively. The first peptide interacts with site 1 through its sTyr41 residue in a manner similar to the pCS molecule. The preceding residues ${ }^{36} \mathrm{FPDGD}^{40}$ close the back of the binding pocket, decreasing the solvent exposed surface area of sTyr41, and provide additional stability through multiple intermolecular interactions (figure 4C). Phe36 and Pro37 pack hydrophobically against Lys51 and Lys52 of LukE. Asp38 forms a salt bridge with LukE Arg290, and Gly39 carbonyl oxygen is hydrogen bonded to Arg101 from site 2 . The $2^{\text {nd }}$ peptide sTyr41 residue interacts with site 2 , in a way that is also similar to $\mathrm{pCS}$, although Arg101 sidechain adopts a different conformation and an additional salt bridge is formed between the sulfate and Lys283 (figure 4E). Other stabilizing interactions include several backbone-backbone hydrogen bonds between Asp40-Lys92, Gly42-Asp90, and Leu45-Phe88, and hydrophobic interactions between Leu45 and Phe88, Ala219 and Arg220 of LukE. 
bioRxiv preprint doi: https://doi.org/10.1101/2021.08.05.455213; this version posted August 6, 2021. The copyright holder for this preprint (which was not certified by peer review) is the author/funder, who has granted bioRxiv a license to display the preprint in perpetuity. It is made available under aCC-BY 4.0 International license.

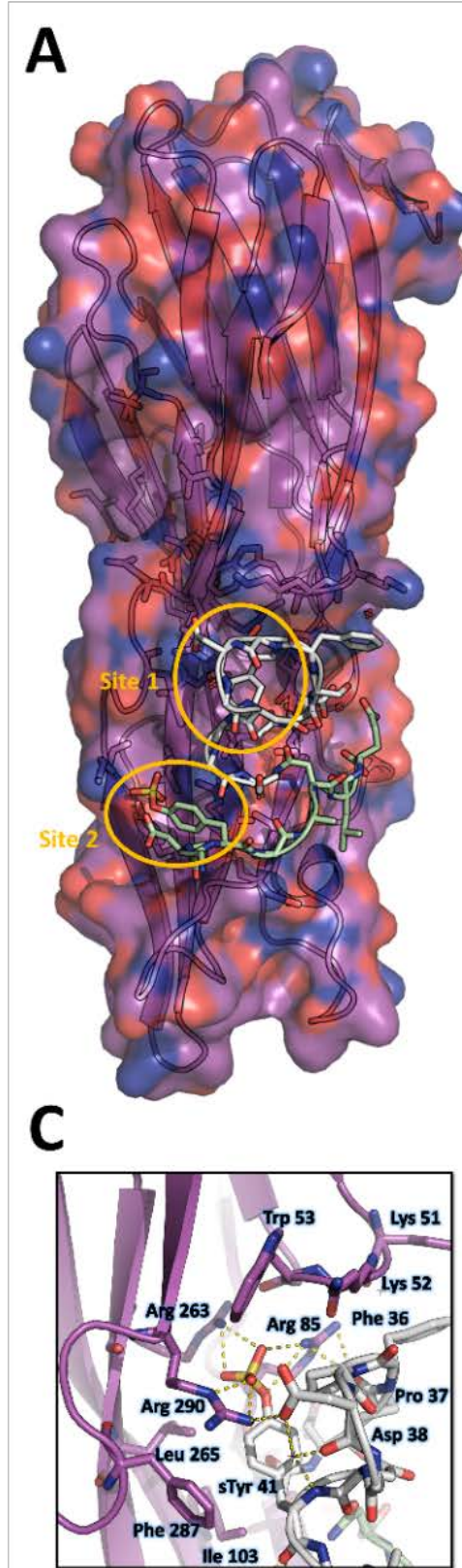

E

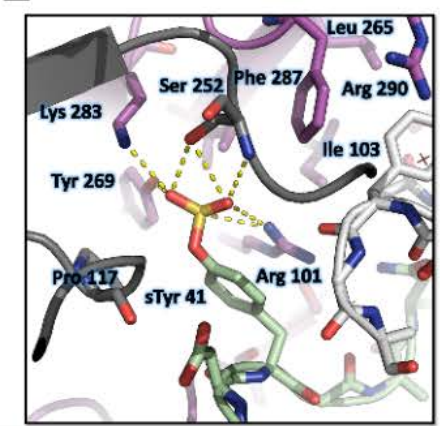

B

D
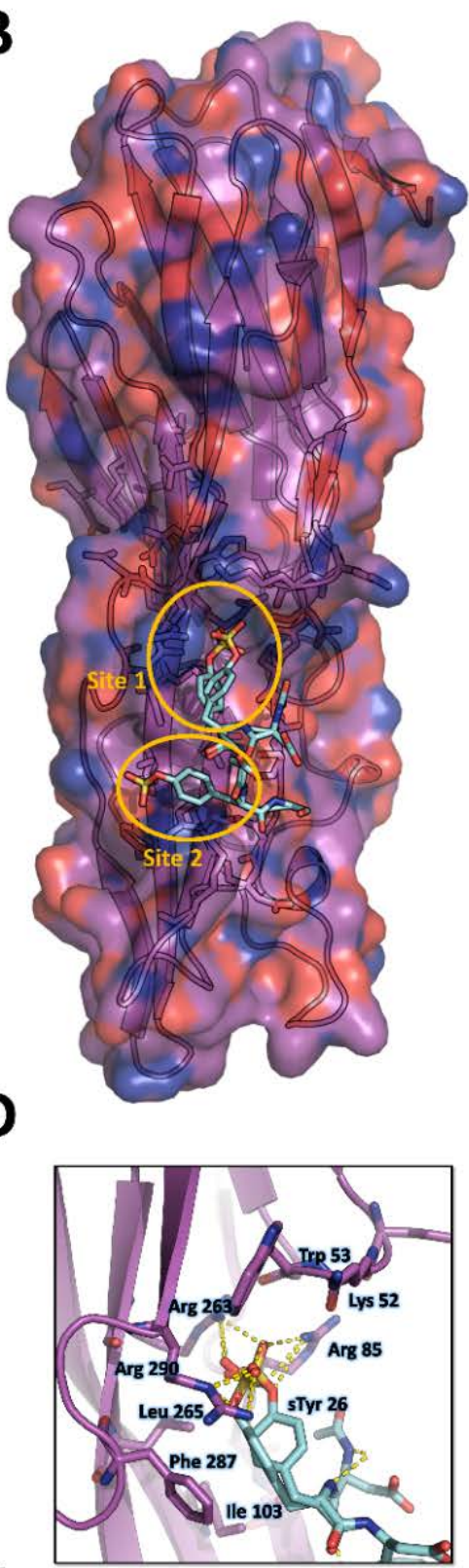

$\mathbf{F}$

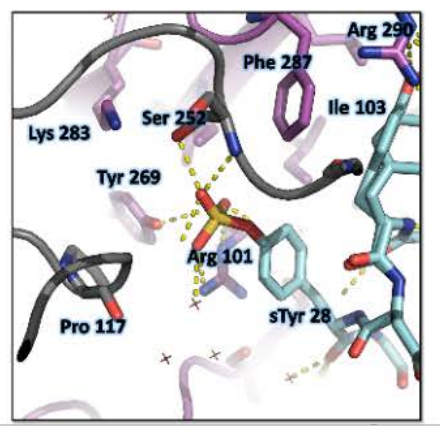

Figure 4: Crystal structures of LukE in complex with ACKR1 peptide ${ }^{34}$ DSFPDGDsYGANLE ${ }^{46}$ and CCR2 peptide ${ }^{25}$ DsYDsYG $^{29}$. A. Overview of the asymmetric unit of the ACKR1 peptidesoaked crystal. Protein is shown in cartoon representation and colored in magenta. The two copies of the peptide with their respective sulfotyrosine residue bound to site 1 and 2 are shown in white and green cartoon and sticks representation. Surrounding protein sidechains are shown as magenta lines. $B$. Overview of the asymmetric unit of the CCR2 peptide-soaked crystal. Protein is shown in cartoon representation and colored in magenta. The CCR2 peptide is shown in cyan cartoon and sticks representation. Surrounding protein sidechains are shown as magenta lines. C and D. Close ups of the sulfotyrosine binding site 1 in the ACKR1 (C) and CCR2 (D) peptide-soaked crystals. The color code is the same as in $A$ and $B$ with peptide and protein sidechains shown in sticks. Polar contacts between ligand and protein are shown as yellow dashed lines. E and F. Close ups of the sulfotyrosine binding site 2 in the ACKR1 (E) and CCR2 (F) peptide-soaked crystals. Protein residues from an interacting symmetry related molecule are shown in grey cartoon and sticks. 
345 The structure of LukE bound to the CCR2 peptide ${ }^{25}$ DsYDsYG $^{29}$ is represented in figure 4B. We found that sTyr26 and sTyr28 of CCR2 respectively bind to site 1 and site 2 through the same interactions observed in the pCS and ACKR1 peptide-bound structures (Fig 4D and F). Two alternate conformations were clearly visible for sTyr26, differing by a rotation of approximately $90^{\circ}$ of the phenyl ring (figure $4 \mathrm{C}$ and figure S4). However, residual electron density was still present after model building and suggested the existence of a third alternate conformation of sTyr26, with its sulfate group interacting at site 2 (figure S4C and D). This third alternate conformation clashes with the sulfate group of sTyr28, suggesting the existence of a minor population of molecules in which sTyr28 unbinds while sTyr26 flips its sidechain to the second site. This indicates that the binding of the doubly sulfated CCR2 peptide is highly dynamic within the crystal.
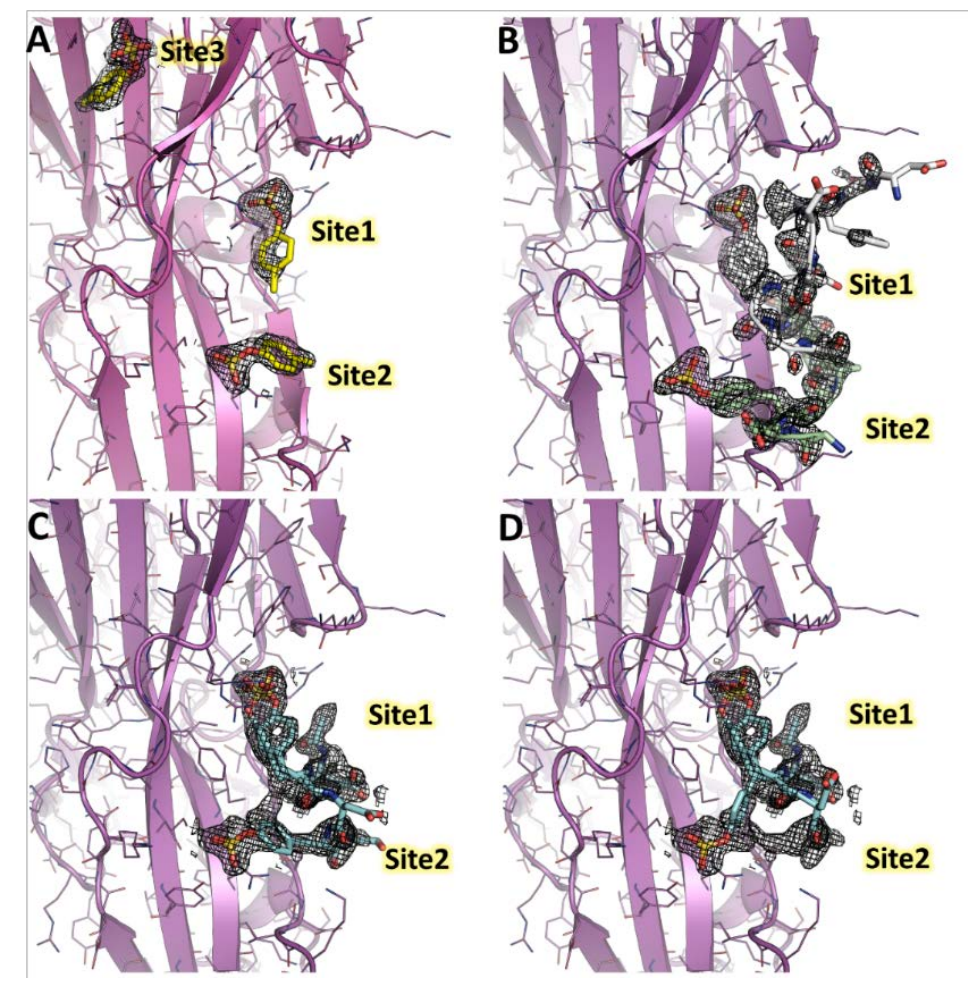

Supplementary figure 4: 2Fo-Fc electron density maps of LukE complexes overlaid with the corresponding models. For each structure, the model is shown in cartoon representation with a color code similar to figure 3,4 and 5. The electron density maps are shown as mesh and contoured at 1.2б. A. 2Fo-Fc map for the p-cresol sulfate-soaked structure. B. 2Fo-Fc map for the ACKR1 ${ }^{34}$ DSFPDGDsYGANLE ${ }^{46}$-soaked structure C. 2Fo-Fc map for the CCR2 ${ }^{25} D s Y D s Y G^{29}$-soaked structure, corresponding to the conformation that was deposited in the PDB. D. Same as in C, but the model was modified to show a third possible alternate conformation of sTyr26, suggesting the peptide is in equilibrium between different bound conformations.

\section{Docking simulations of LukE onto ACKR1 and CCR5}

In order to frame these crystallographic results into a more global context, we set out to build computational models of the full-length ACKR1-LukE and CCR5-LukE complexes. A wealth of mutagenesis data is available in the literature that provides partial information about 
interacting residues for the ACKR1-LukE (Spaan, Reyes-Robles et al. 2015, Peng, Takeshita et al. 2018, Vasquez, Lubkin et al. 2020) and CCR5-LukE complexes (Reyes-Robles, Alonzo et al. 2013, Tam, Schultz et al. 2016). The regions of LukE that are potentially implicated in receptor interaction based on previous work and on the sulfopeptide-bound structures were mapped onto the structure of LukE (figure S5A), revealing a contiguous interaction surface.

The task of docking LukE onto ACKR1 and CCR5 was performed in 2 steps: in the first step, the toxin was docked onto the experimental structures (for CCR5) or a theoretical model (for ACKR1) without the $\mathrm{N}$-terminal region of the receptor, using the information-driven docking software HADDOCK. Available mutagenesis data were encoded into Ambiguous Interaction Restraints (AIRs) by HADDOCK, providing relatively strong constraints on the orientation of the bound toxin (see methods). In the $2^{\text {nd }}$ step, the tyrosine-sulfated $\mathrm{N}$-terminal region of the receptor was added to the best model of each complex, and multiple MD simulations were performed in order to simultaneously dock the $\mathrm{N}$-terminal region of the receptor onto the toxin and assess the stability of the docking pose.

Figure S5 B and C show the HADDOCK (energy) score versus interface R.M.S.D. (iRMSDrelative to the best scoring model) for the best ACKR1-LukE and CCR5-LukE docking runs, revealing in both cases a deep energy funnel corresponding to the best cluster. The best scoring ACKR1-LukE and CCR5-LukE clusters are also the largest ones with 44 and 67 members, respectively. The best scoring pose for each complex is shown in figure S5 D and E, after addition of the missing $\mathrm{N}$-terminal residues and prior to $\mathrm{MD}$ simulations. The relative orientation of LukE in each complex is somewhat different, with CCR5-bound LukE forming a more acute angle with the membrane plane compared to the ACKR1-LukE pose (figure S5 D and E). However, in both cases LukE loop 4 inserts into the orthosteric pocket and interacts with ACKR1 eCL3 and TMs residues, while loop 3 interacts with eCL2. Importantly, the position of TM1 and the N-terminal region of the receptor for both toxin-receptor pairs seem compatible with sulfotyrosine interactions at site 1 and/or 2 observed in crystal structures. Indeed, ACKR1 sTyr 41 is located 10 residues upstream of Cys51 that marks the beginning of TM1, while the potentially sulfated tyrosines of CCR5 (sTyr3, sTyr10, sTyr 14 and sTyr 15) are located 5 to 17 residues upstream of the equivalent Cys20 at the top of CCR5 TM1. This short distance between TM1 and the sulfated tyrosines, combined with the known location of site 1 and 2 on LukE, provide an additional constraint on the possible orientations of the toxin, further suggesting the correctness of the identified docking poses.

400

401

402

403

404

405

406

407

408

409
In order to assess the stability of these poses and dock the $\mathrm{N}$-terminal region of both receptors onto LukE, we ran multiple MD simulations starting from the equilibrated models shown in figure S5 D and E (see methods). Only the functionally critical Tyr41 was sulphated in the ACKR1-LukE model, as the other sulfotyrosine in position 33 was shown to have very limited impact on LukED activity (Spaan, Reyes-Robles et al. 2015). In the case of CCR5, no information is available about which of the potentially sulfated tyrosines are important for binding LukED, although all 4 tyrosines are heterogeneously sulfated in vivo, and sulfation is known to affect binding to other CCR5 ligands (Farzan, Mirzabekov et al. 1999, Jen, Moore et al. 2009). For these reasons, all 4 tyrosines were sulfated in our CCR5-LukE model. 

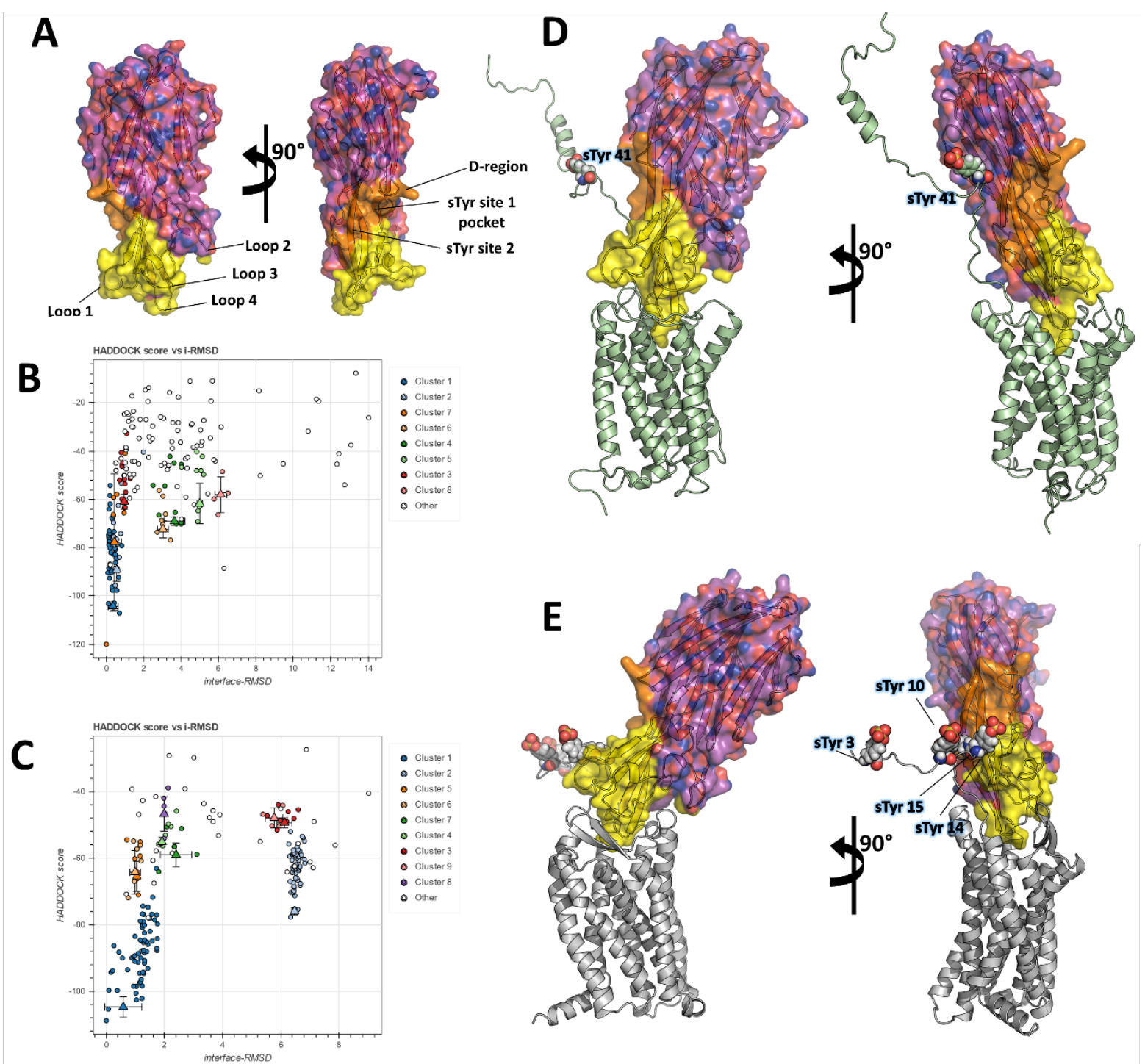

Supplementary figure 5: Computational docking of ACKR1-LukE and CCR5-LukE. A Mapping of the LukE residues potentially involved in receptor binding. LukE is shown in semi-transparent surface and cartoon representation and coloured in magenta. Site $1 \& 2$ sulfotyrosine binding sites are coloured in orange. Other residues that are potentially involved in receptor binding are coloured in yellow based on previous mutagenesis work (Nariya and Kamio 1997, Reyes-Robles, Alonzo et al. 2013, Tam, Schultz et al. 2016, Peng, Takeshita et al. 2018, Vasquez, Lubkin et al. 2020). B and C. HADDOCK score versus interface R.M.S.D. (iRMSD- relative to the best scoring model) plots for the best ACKR1-LukE (B) and CCR5-LukE (C) docking runs. The best cluster identified by HADDOCK (cluster 1) is shown in blue. D an E. ACKR1-LukE (D) and CCR5-LukE (E) top scoring models selected from HADDOCK simulations, after addition of the missing $\mathrm{N}$-terminal region (residues 8 to 50 of ACKR1 and residues 1 to 19 of CCR5), energy minimization and MD equilibration. The receptor is shown in cartoon representation and colored in pale green (ACKR1) or light gray (CCR5). Sulfated tyrosines are shown as spheres. binding pocket for ACKR1 N-terminal peptide.

A summary of the information extracted from MD simulations is shown in table 2. For both complexes, we monitored the number of inter-chain hydrogen bonds ( $H$-bonds), buried surface area (BSA) and interface R.M.S.D. (iRMSD) relative to the starting model, as these 
parameters were recently shown to be useful in discriminating native from non-native docked models by MD (Jandova, Vargiu et al. 2021). We additionally outputted the time evolution of distances involving receptor sulfotyrosines and LukE site 1 and 2 residues (Figure S6/S8), and visually inspected models for qualitative agreement with available mutagenesis data. The best models for ACKR1-LukE and CCR5-LukE were taken from the stable region of the trajectory showing the highest $\mathrm{H}$-bonds, highest BSA and most stable iRMSD, while being consistent with all experimental observations (Figure S6/S8 and Table 2).

\begin{tabular}{|c|c|c|c|c|c|c|}
\hline & $\begin{array}{c}\text { Total } \\
\text { simulation } \\
\text { time }(\mu \mathrm{s})\end{array}$ & $\langle i R M S D>(n m)$ & $<\mathrm{BSA}>\left(\mathrm{nm}^{2}\right)$ & $\begin{array}{c}\text { <number of } \mathrm{H}- \\
\text { bondss> }\end{array}$ & $\begin{array}{c}\text { Site } 1 \\
\text { sulfotyrosine } \\
\text { interaction }\end{array}$ & $\begin{array}{c}\text { Site } 2 \\
\text { sulfotyrosine } \\
\text { interaction }\end{array}$ \\
\hline \multicolumn{7}{|c|}{$\begin{array}{l}\text { ACKR1-LukE MD } \\
\text { trajectory }\end{array}$} \\
\hline 1 & 1.44 & $0.49(0.04)$ & $45.5(6.4)$ & $20.0(4.9)$ & sTyr41 & Asn44/Glu46 \\
\hline 2 & 0.84 & $0.56(0.05)$ & $39.7(7.3)$ & $18.9(4.6)$ & sTyr41 & Glu46 \\
\hline 3 & 1.21 & $0.41(0.04)$ & $49.5(6.8)$ & $21.3(4.8)$ & Not interacting & Not interacting \\
\hline AWH & 2.15 & $0.55(0.07)$ & $41.8(5.9)$ & $14.0(4.0)$ & sTyr41* & Glu46* \\
\hline \multicolumn{7}{|c|}{$\begin{array}{l}\text { CCR5-LukE MD } \\
\text { trajectory }\end{array}$} \\
\hline 1 & 1.42 & $0.37(0.03)$ & $38.9(5.3)$ & $18.5(4.1)$ & sTyr10 & sTyr14 \\
\hline 2 & 1.22 & $0.49(0.05)$ & $35.4(5.8)$ & $16.0(4.5)$ & sTyr10 & sTyr15 \\
\hline 3 & 1.14 & $0.46(0.05)$ & $36.2(3.4)$ & $15.5(3.7)$ & sTyr10 & sTyr15 \\
\hline
\end{tabular}

*Interactions observed in the global energy minimum basin of the PMF.

Table 2: Summary of MD simulations. <> denotes average over simulation time. Values in parentheses correspond to standard deviation. Lines corresponding to the best MD trajectories (that were used to make Figure 5 and 6) are shown in bold.

Representative MD snapshots of the best ACKR1-LukE model are shown in figure 5A. The interactions between ACKR1 orthosteric pocket and LukE divergent loops initially rearranged during the first $200 \mathrm{~ns}$ of $\mathrm{MD}$ and then remained stable for the duration of the simulation, as shown by the iRMSD plateau at $\sim 5 \AA$ (figure S6B). LukE Arg275 located at the tip of loop4 inserted into the orthosteric pocket, forming a stable salt bridge with ACKR1 Asp263 (Figure 5D). The disulfide bonded Cys-51 and Cys276 interacted with a large hydrophobic surface patch of LukE involving Tyr96, Thr99, Thr271, Phe273 and Tyr 279 located in loops 1 and 4 (Figure 5C). Consistent with our modeling results, ACKR1 Cys51 was previously identified by mutagenesis as critical for LukED intoxication of erythrocytes (Spaan, Reyes-Robles et al. 2015), as well as LukE loops 1, 3 and 4 (Peng, Takeshita et al. 2018, Vasquez, Lubkin et al. 2020). The N-terminal region of ACKR1 quickly attached to the toxin surface during MD (Figure 5B), leading to an increase in the complex BSA and number of $\mathrm{H}$-bonds (figure S6C and D). ACKR1 sTyr 41 bound to site 1 arginines within less than 100ns of simulation in 2 out of 3 trajectories, while Glu46 interacted with site 2 residues Tyr269 and Lys283 (Figure 5B and S6 EFG). The remainder of ACKR1 N-terminal region included in our model (residues 8-40) adopted various bound conformations over time in different MD trajectories, without converging to a single bound state. 


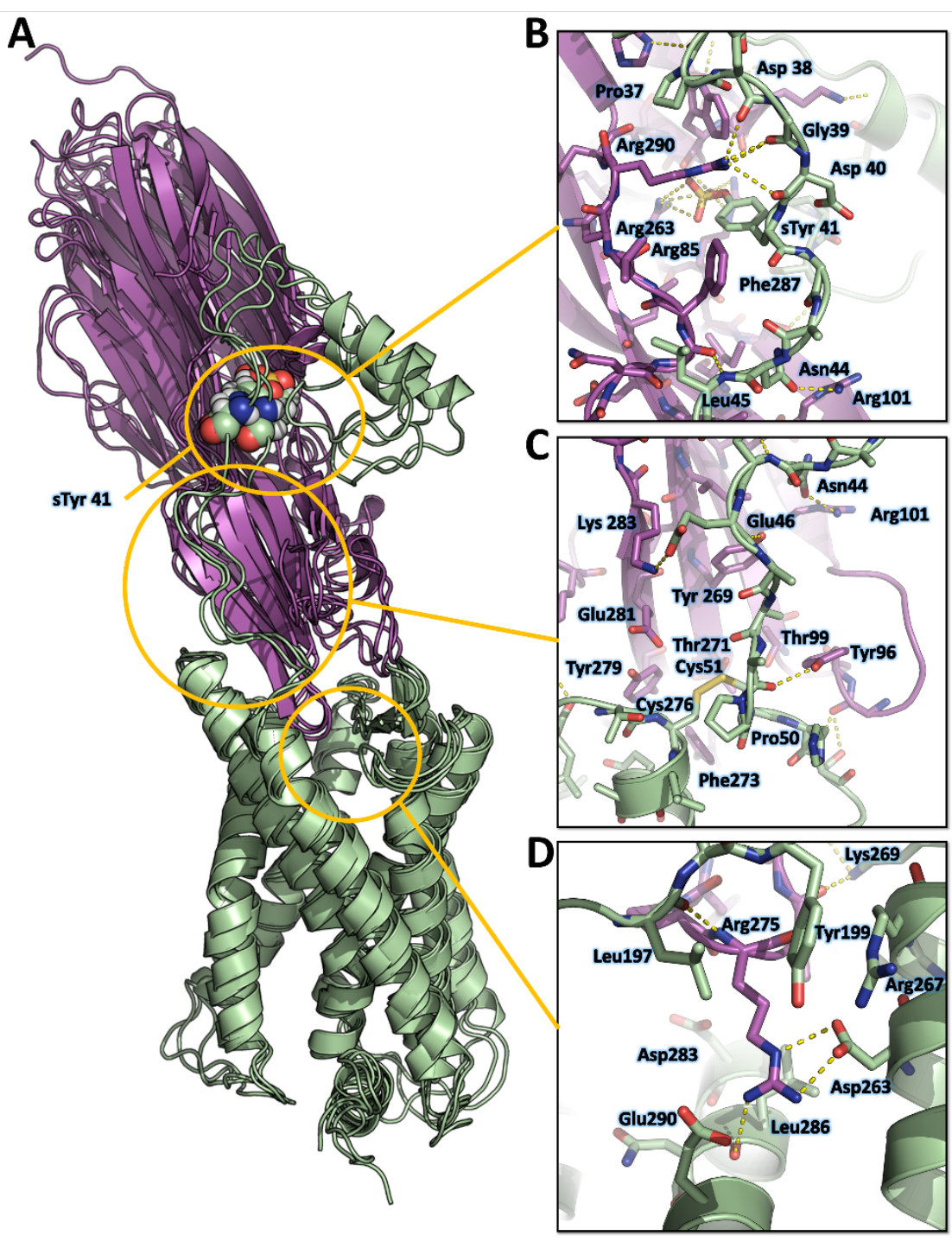

Figure 5: Computational model of the ACKR1-LukE complex extracted from MD simulations. A. Superimposed MD snapshots extracted from the last 300 ns of simulation (3 snapshots taken at $100 \mathrm{~ns}$ interval). The receptor and toxin are shown in cartoon representation and colored in pale green and magenta, respectively. ACKR1 sTyr 41 is shown as spheres. B, C and D. Close ups of the interactions observed in the proposed model at the sulfotyrosine binding site 1 (B), sulfotyrosine binding site 2 (C), and the orthosteric pocket (D). Interacting residues are displayed as sticks. The color code is the same as in $\mathrm{A}$.

Although these simulation results suggest that ACKR1 sTyr 41 interacts with LukE site 1, classical MD simulations have a known tendency to kinetically trap proteins in their closest energy minimum relative to the starting conformation (Bernardi, Melo et al. 2015). In order to assess the relative stability of sTyr 41 binding to site 1 versus site 2 (both of which were observed crystallographically), we performed an enhanced sampling MD simulation using the accelerated weight histogram (AWH) method (Lindahl, Lidmar et al. 2014). This method enables the calculation of potential of mean force (PMF) profiles along (a) chosen reaction coordinate(s), and functions by applying a time-dependent biasing potential that flattens free energy barriers along the reaction path. In the case of ACKR1-LukE, a two-dimensional reaction coordinate was selected to report on site 1 (sTyr 41 - Arg263 distance) and site 2 
bioRxiv preprint doi: https://doi.org/10.1101/2021 08.05.455213: this version posted August 6 . 2021. The copvriaht holder for this preprint (which was not certified by peer review) is the author/funder, who has granted bioRxiv a license to display the preprint in perpetuity. It is made available under aCC-BY 4.0 International license.

(sTyr41 - Tyr269 distance) interactions. After extensive sampling of the reaction coordinate

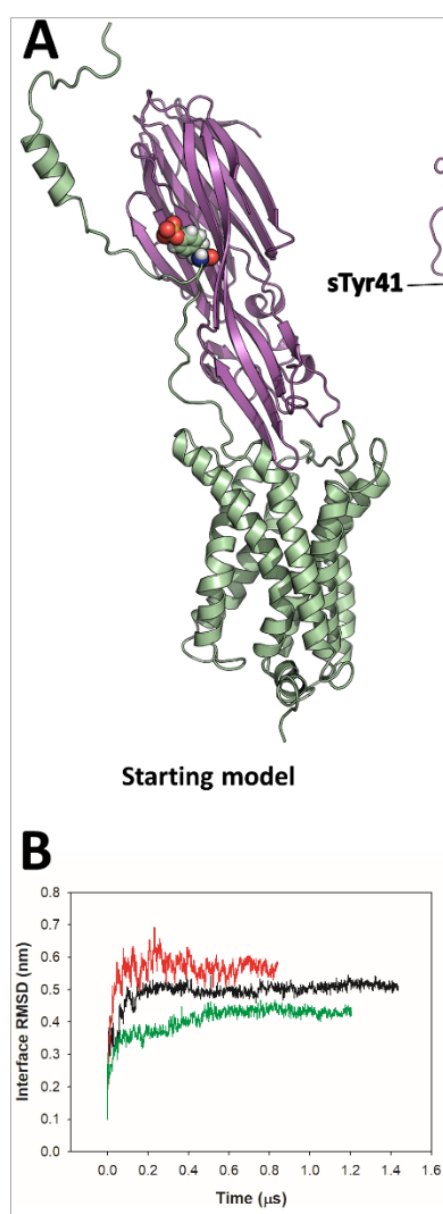

\section{E}

End model 1

\section{C}
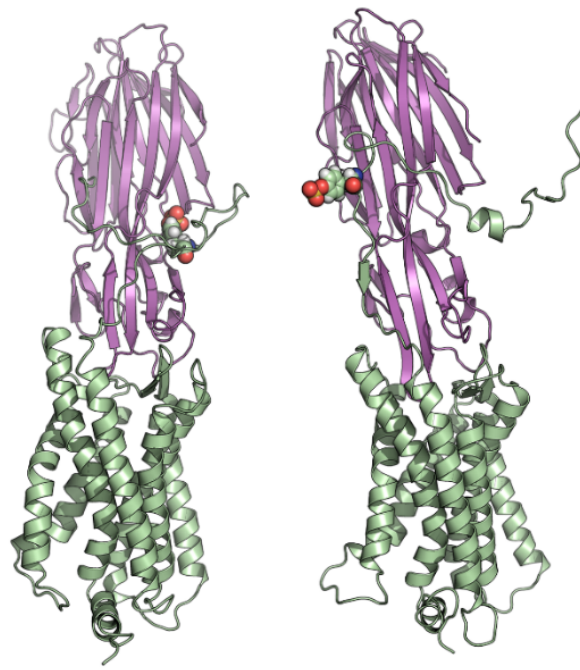

End model 2

End model 3

\section{D}
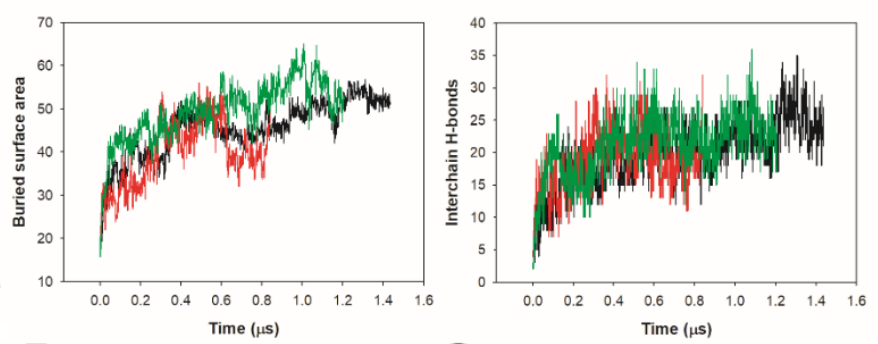

G
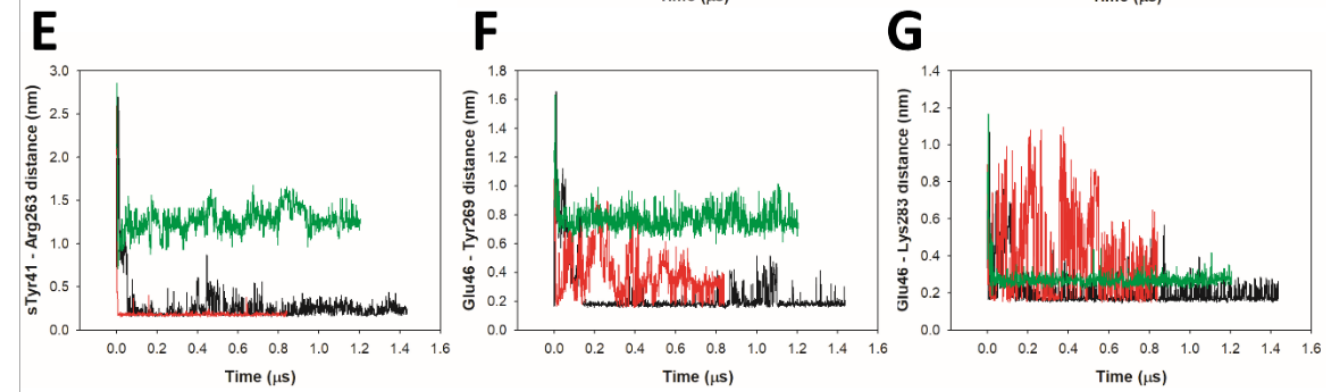

Supplementary Figure 6: Analysis of ACKR1-LukE MD simulations. A. Starting model and end model snapshots for each of the 3 independent CCR5-LukE MD trajectories. B to G. Time-dependent properties of the system extracted from MD simulations for trajectories 1, 2 and 3 (black, red and green lines, respectively): RMSD of the interface residues relative to the starting model (B), buried surface area in the complex (C), number of interchain hydrogen bonds (D), sTyr41 - Arg263 minimum distance (E), and Glu46 - Tyr269 minimum distance (F). Interface residues for iRMSD calculation were defined based on the starting structure using a $1 \mathrm{~nm}$ cutoff, and calculation 


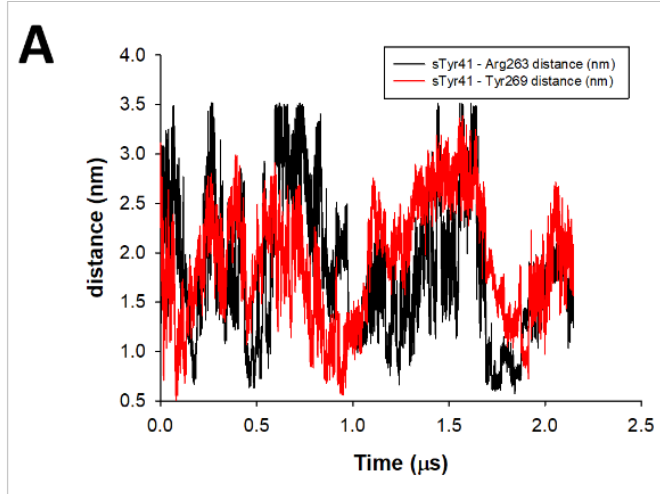

\section{C}

B
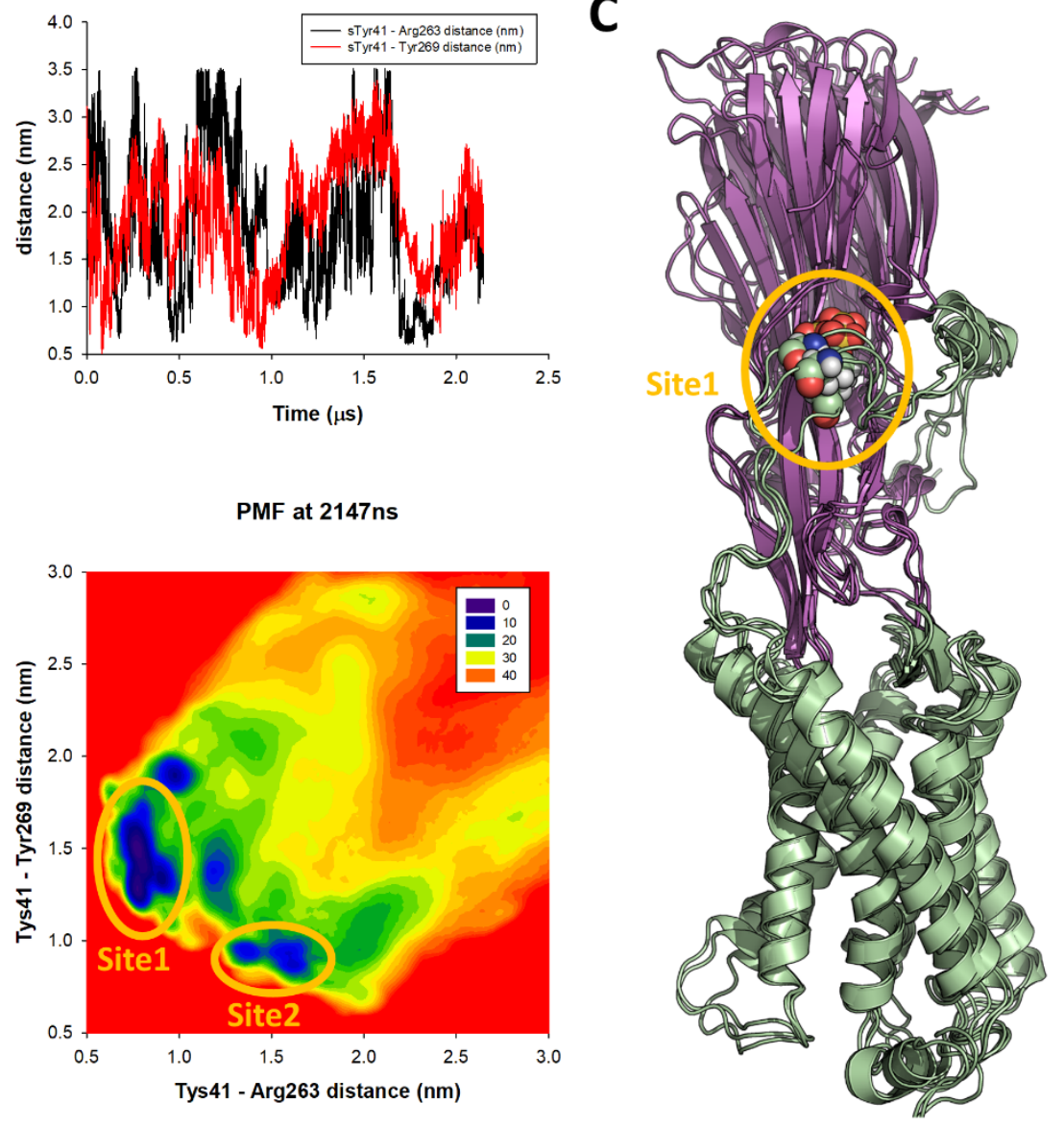

Figure 6: AWH MD simulation of the ACKR1-LukE model. A. sTyr 41 - Arg263 (site 1 - black line) and sTyr41 Tyr269 (site 2 - red line) center of mass (COM) distance as a function of simulation time, highlighting the extensive sampling of these variables during the AWH simulation. B. Final potential of mean force (PMF) profile calculated at $\mathrm{t}=2.147 \mu \mathrm{s}$. The heat map represents free energies in $\mathrm{kJ} / \mathrm{mol}$. The energy wells corresponding to site 1 and site 2 interactions are circled in orange. C. 3 snapshots extracted from the AWH MD simulation at $\mathrm{t}=$ $1.730,1.740$ and $1.750 \mu$ s are superimposed, which sample the global energy minimum of the PMF.

Movie S1: Time evolution of the ACKR1-LukE model conformation and estimated PMF during AWH MD. The movie contains 215 frames from $t=0$ to $t=2.14 \mu s$ (with a 10 ns timestep). Note the sampling of "site 1 " free recognizes both site 1 and 2 .

Representative MD snapshots of the best CCR5-LukE model are shown in figure 6A. The toxinreceptor relative orientation and interations between LukE loops and CCR5 orthosteric pocket remained mostly stable during MD with iRMSD values of $0.4-0.5 \mathrm{~nm}$ (figure S7A and B). The observed interactions were consistent with information available in the literature (Reyes- 
(Figure 6D). After initial rearrangements, LukE Arg275 from loop 4 formed stable ionic interactions with CCR5 Asp276 and Glu283 located in the upper part of TM7 (Figure 6D). Similar to the ACKR1-Luke model, the N-terminal region of CCR5 rapidly docked itself onto the toxin surface in a process most likely driven by long range electrostatics, creating interactions with LukE loop 1 and with the sulfotyrosine binding sites (Figure 6B and C). In all $3 \mathrm{MD}$ trajectories, CCR5 sTyr 10 bound to LukE sulfotyrosine site 1 after 100-400 ns of simulation time (figure $6 \mathrm{C}$ and figure S7E). Binding of sTyr 14 or 15 to sulfotyrosine site 2 were also observed in all trajectories, however this interaction was more dynamic, possibly because both residues were sulfated and able to interact with LukE site 2 residues (figure 6B and figure S7F and G). The first $\mathrm{N}$-terminal residues of CCR5 (residues 1-9) adopted various conformations in different trajectories without converging to a stable bound state.

B

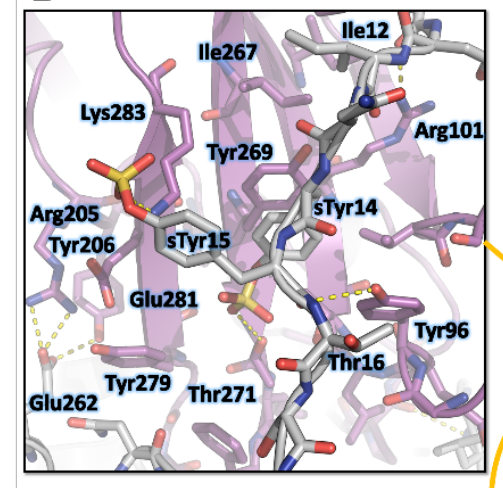

D

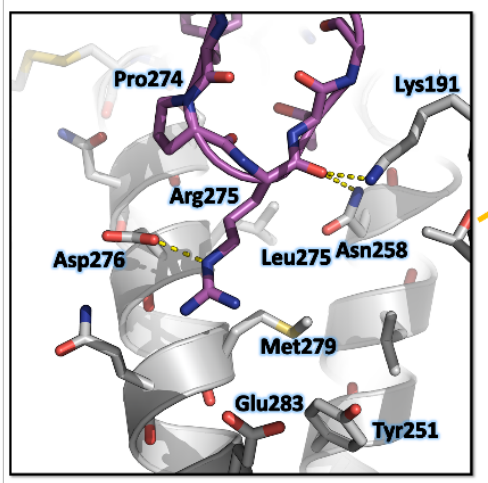

A

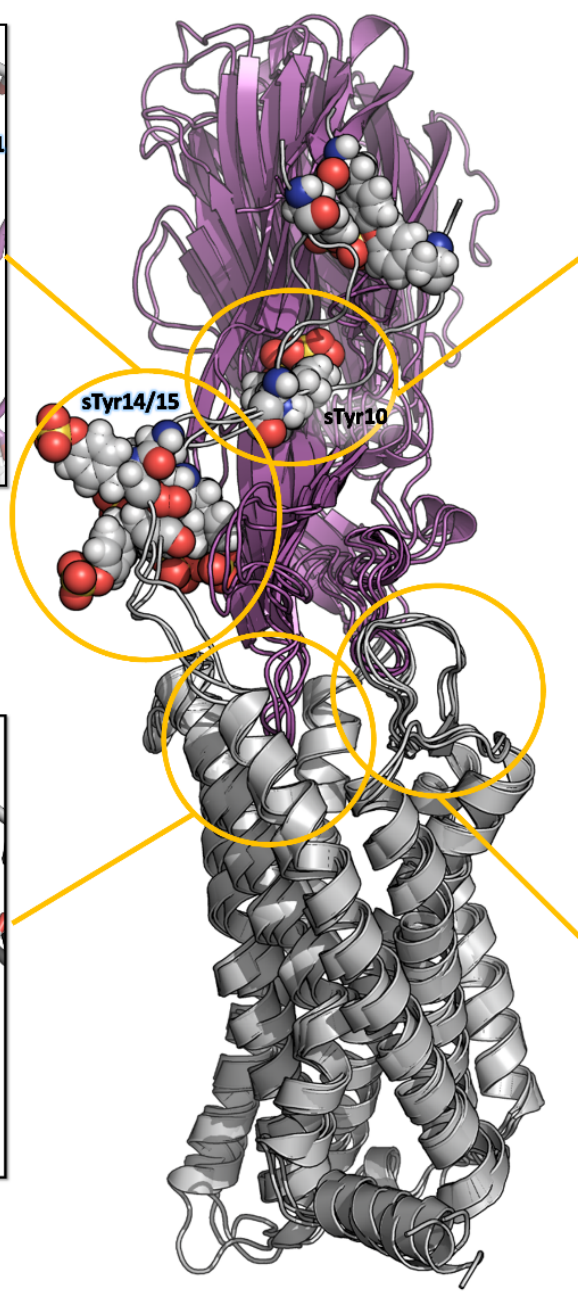

C

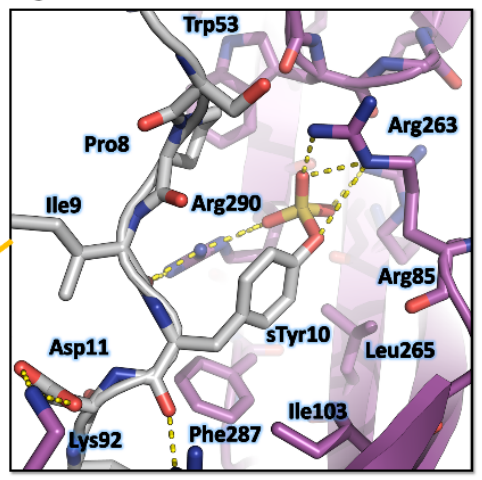

E

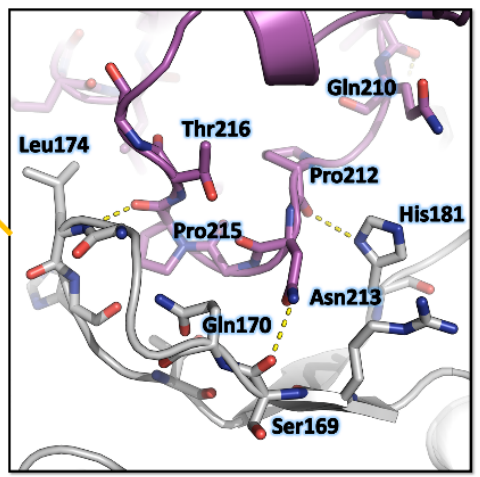

Figure 7: Computational model of the CCR5-LukE complex extracted from MD simulations. A. Superimposed MD snapshots extracted from the last 300ns of simulation (3 snapshots taken at $100 \mathrm{~ns}$ interval). The receptor and toxin are shown in cartoon representation and colored in light gray and magenta, respectively. CCR5 STyr residues are shown as spheres. $B, C D$ and $E$. Close ups of the interactions observed in the proposed model at the sulfotyrosine binding site 2 (B), sulfotyrosine binding site 1 (C), orthosteric pocket (D) and CCR5 eCL2/LukE loop3 region (E). Interacting residues are displayed as sticks. The color code is the same as in $A$. 


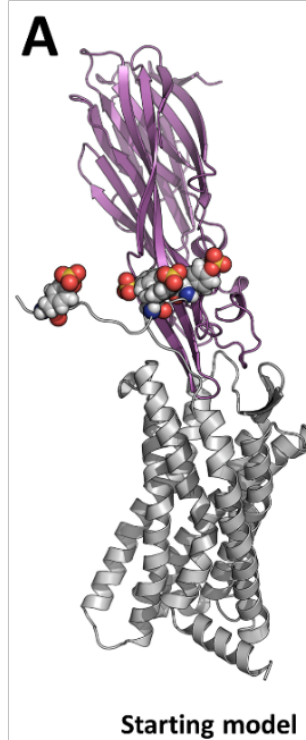

B

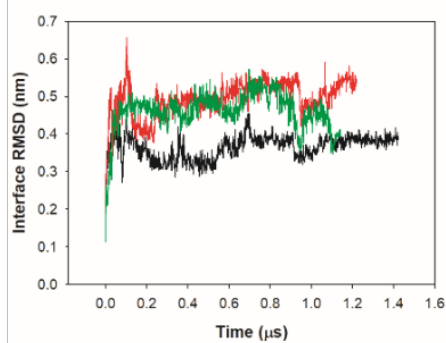

E

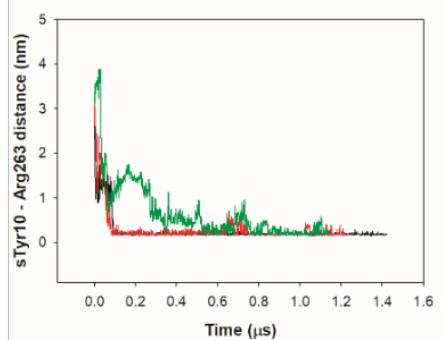

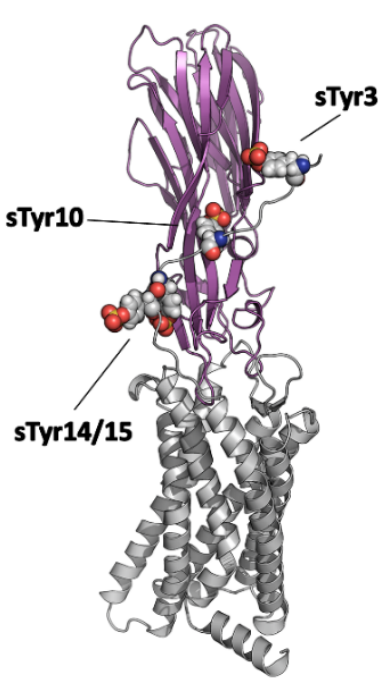

End model 1

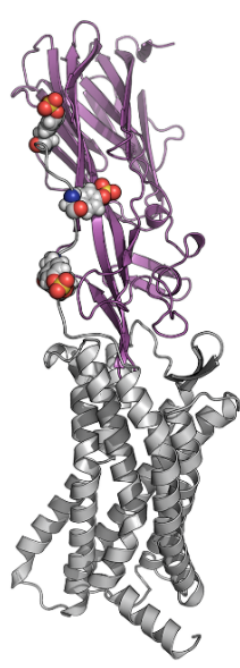

End model 2

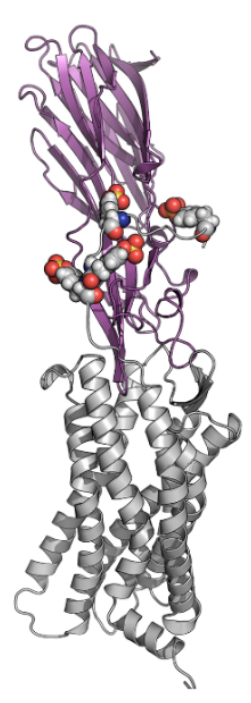

End model 3

D
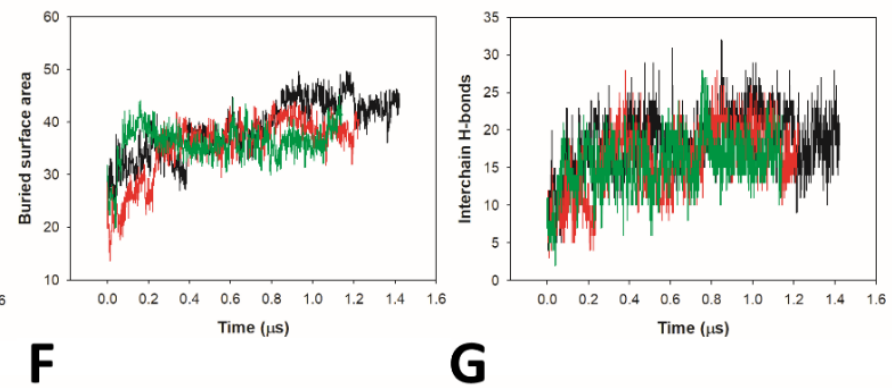

G
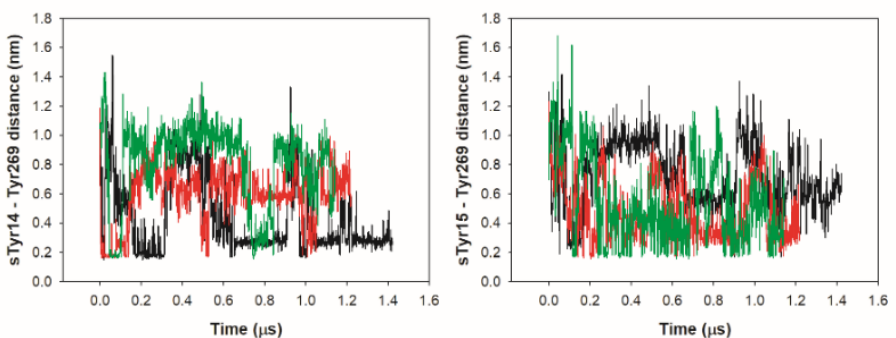

Supplementary Figure 7: Analysis of CCR5-LukE MD simulations. A. Starting model and end model snapshots for each of the 3 independent CCR5-LukE MD trajectories. B to G. Time-dependent properties of the system extracted from MD simulations for trajectories 1, 2 and 3 (black, red and green lines, respectively): RMSD of the interface residues relative to the starting model (B), buried surface area in the complex (C), number of interchain hydrogen bonds (D), sTyr10 - Arg263 minimum distance (E), sTyr14 - Tyr269 minimum distance (F), and sTyr15 Tyr269 minimum distance (G). Interface residues for iRMSD calculation were defined based on the starting structure using a $1 \mathrm{~nm}$ cutoff, and calculation was performed on $\mathrm{C} \alpha$ atoms.

\section{Discussion}

The present study provides an in-depth characterization of the structure of monomeric LukE and of its ability to bind the chemokine receptors ACKR1, CCR2 and CCR5. TR-FRET competition assays indicated that LukE can displace bound CCL2/CCL5 from their cognate receptors, confirming significant overlap between the chemokine and toxin binding surfaces on the receptors. Surprisingly, LukE competed with CCL2 for CCR2 binding with a slightly better IC50 
than for the other receptors, suggesting a strong interaction between CCR2 and LukE. This indicates that even though LukED is unable to lyse CCR2-expressing cells (Tam, Schultz et al. 2016), LukE can still prevent CCR2 signalling. Such competitive inhibition might exist for other chemokine receptors bearing sulfotyrosines in their $\mathrm{N}$-terminus, and could play a role in SA infections by modulating inflammatory responses.

Based on the information available in the literature, it appeared that sulfotyrosine recognition may constitute a common feature of leukotoxin-GPCR interactions, which may in part explain their broad receptor specificity. We thus sought to obtain high resolution structural information regarding this type of interactions. The crystal structure of LukE in complex with p-cresyl sulfate identified 3 potential sulfotyrosine binding sites, 2 of which were also observed in the LukE structures in complex with peptides derived from the $\mathrm{N}$-terminal region of ACKR1 and CCR2. We then used protein-protein docking and MD simulations to propose models of the ACKR1-LukE and CCR5-LukE complexes that are consistent with the crystallographic data and with previous mutagenesis data (Reyes-Robles, Alonzo et al. 2013, Spaan, Reyes-Robles et al. 2015, Tam, Schultz et al. 2016, Peng, Takeshita et al. 2018, Vasquez, Lubkin et al. 2020). Our work demonstrates that site 1 is used to bind ACKR1 sTyr 41, CCR2 sTyr 26 and CCR5 sTyr 10, while site 2 can accommodate an additional downstream sulfotyrosine such as CCR5 sTyr 14 or 15, or CCR2 sTyr 28.

\section{Conservation of the sulfotyrosine binding sites among GPCR-binding leukotoxins}

Site 1 is widely conserved across leukotoxin S components, and the 3 arginines ( $\operatorname{Arg} 85,263$ and 290 of LukE) coordinating the tyrosine sulfate are strictly conserved in HIgA, HIgC and lukSPV (figure S8 BDEG). The 3 hydrophobic residues that are in direct interaction with the sulfotyrosine phenyl ring (Ile103, Leu265, Phe287 of LukE) show more variability, with Leu265 mutated to Met in HIgA, and both Leu265 and lle103 changed to Met and Arg in HIgC and LukS-PV. This high level of conservation strongly suggests that all 4 toxins use site 1 to recognize sulfated tyrosines in their respective receptors. These receptors contain sulfotyrosines within a YXY (CXCR2, CCR2), YXXY (C5aR1, C5aR2) or YXXXY (CCR5) motif, except for ACKR1 and CXCR1 that possess isolated sulfotyrosine residue(s) (figure S8A). 
bioRxiv preprint doi: https://doi.org/10.1101/2021.08.05.455213; this version posted August 6, 2021. The copyright holder for this preprint (which was not certified by peer review) is the author/funder, who has granted bioRxiv a license to display the preprint in perpetuity. It is made available under aCC-BY 4.0 International license.

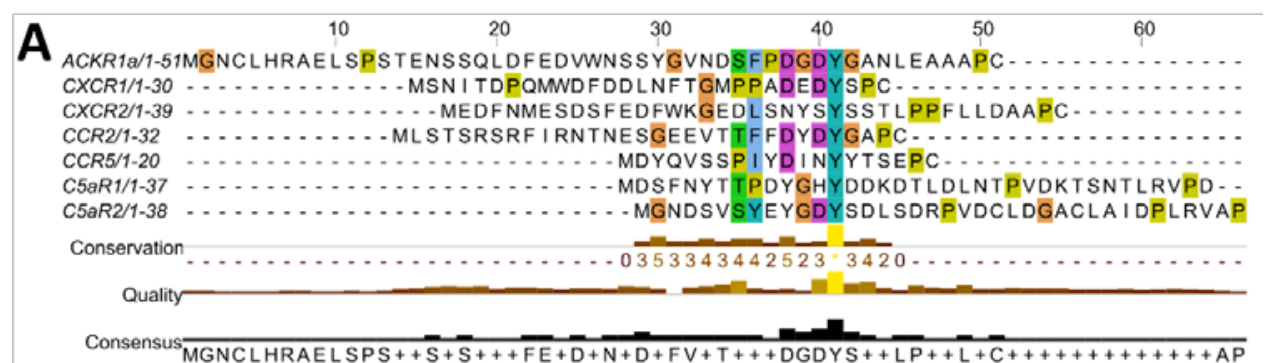

\section{S -components}

B

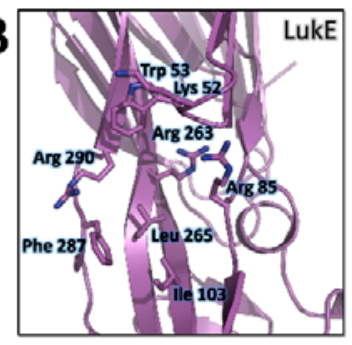

D
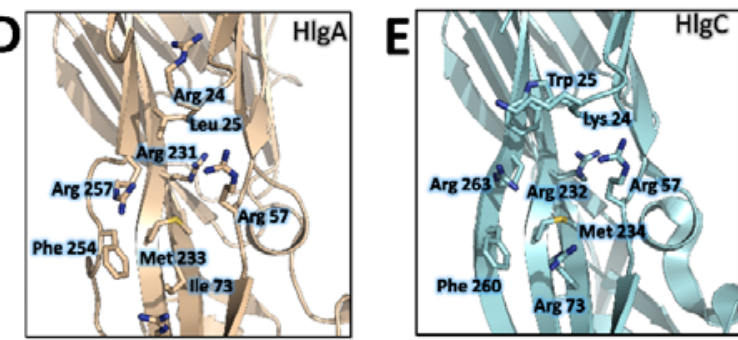

G

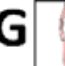

I

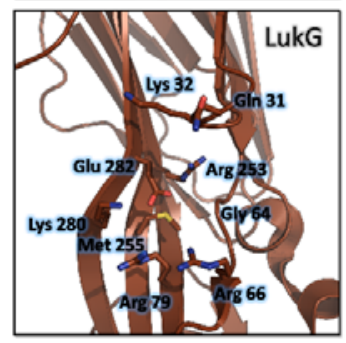

F -components
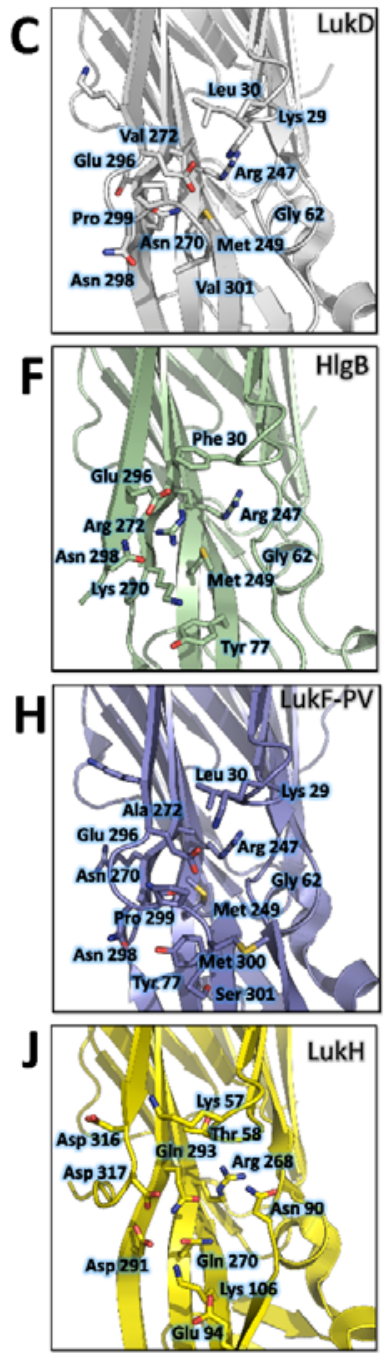

Supplementary Figure 8: Conservation of the sulfotyrosine sites in the $\mathrm{N}$-terminal region of leukotoxins receptors and structural alignment of Staphylococcus aureus bicomponent pore forming toxins in the sulfotyrosine binding region (site 1). A. manual sequence alignment of the sulfotyrosine motifs in the $\mathrm{N}$-terminal region of the bicomponent pore forming toxin receptors. $B$ to J. Comparison of sulfotyrosine site 1 residues in SA bi-component leukotoxin $x$-ray structures. B. LukE apo1. C. LukD (PDB ID 6U2S, (Liu, Kozhaya et al. 2020)). D. HIgA (PDB ID 2QK7, (Roblin, Guillet et al. 2008)). E. HlgC (PDB ID 4P1X, (Yamashita, Sugawara et al. 2014)). F. HIgB (PDB ID 3LKF, (Olson, Nariya et al. 1999)). G. LukS-PV (PDB ID 1T5R, (Guillet, Roblin et al. 2004)). H. LukF-PV (PDB ID 1PVL, (Pedelacq, Maveyraud et al. 1999)). I and J. LukG and lukH (PDB ID 5K59, (Badarau, Rouha et al. 2016)). 
Consistent with these observations, the post-translational modification pathways involved in

585

586

587

588

589

590

591

592

593

594

595

596

597

598

599

600

601

602

603

604

605

606

607

608

609

610

611

612

613

614

615

616

617

618

619

620

621

622

623 the sulfation of the leukocidin receptors were recently found to impact on $\mathrm{HIgAB}, \mathrm{HIgCB}$, LukED, and PVL induced cytotoxicity, most likely through reduced $\mathrm{S}$ component binding (Tromp, Van Gent et al. 2020). In addition, ACKR1 Tyr41 to Ala mutation was shown to nearly abolish LukED- and HIgAB-induced hemolysis in HEK cells (Spaan, Reyes-Robles et al. 2015), while ACKR1 Tyr41 to Phe mutation caused a 10 fold increase in HIgAB EC50 required to dissociate ACKR1-bound Gai subunit, as measured by BRET (Grison, Leyrat et al. 2021). LukSPV binding to C5aR1 was shown to require tyrosine sulfation as well, with a measured affinity of $127 \mathrm{nM}$ (SD $\pm 17 \mathrm{nM}$ ) between lukS-PV and the receptor N-terminal peptide by ITC (Spaan, Henry et al. 2013). Interestingly, a bound sulfate ion is also visible at LukS-PV site 1 in the PVL crystal structure reported in (Liu, Kozhaya et al. 2020) (PDB CODE 6U3Y). Taken together, these data indicate that the sulfotyrosine - site 1 interactions observed in this study play a critical role in receptor recognition by S-components.

Site 2 is only conserved in LukE and HIgA, and likely confers to these toxins the ability to bind the second sTyr of the motif in the N-termini of CCR2, CCR5, and CXCR2. Divergence in site 2 residues for $\mathrm{hlgC}$ and LukS-PV indicates that these toxins might use a different set of residues to bind the second Y in C5aR1 and C5aR2 YXXY motif.

Contrary to $\mathrm{S}$ components, F-components show very poor conservation of site 1 , except perhaps for $\mathrm{HIgB}$, in which 3 out of 6 sulfotyrosine-interacting residues are conserved with $\mathrm{HIgA} / \mathrm{C}$ and lukS-PV (Figure S8 B to J). Given the recently described interaction between ACKR1 and HIgB (Grison, Leyrat et al. 2021), it is tempting to speculate that these residues might play a role in receptor recognition, although further experiments will be necessary to (in)validate this hypothesis. Intriguingly, the Arg residue sitting at the bottom of site 1 pocket (LukE Arg263) is conserved in all toxins, including the more distantly related lukGH. Nevertheless, $F$ components harbour a C-terminal extension of their last $\beta$ strand with an exposed acidic residue (for example LukD Glu296) that can neutralize the positively charged Arg (Figure S8 CFHJ).

\section{Implications of the proposed ACKR1- and CCR5-LukE models for receptor binding, conformational changes and subsequent pore formation}

In this study, we used computational docking and MD simulations to produce plausible models of the ACKR1- and CCR5-Luke complexes that are consistent with all available experimental data. During most MD simulations, we observed fast binding of ACKR1 sTyr 41 or CCR5 sTyr 10 to LukE Arg263 of site 1 (figure S6E and S8E), with an inter-residue distance going from 3 $\mathrm{nm}$ to $\sim 2 \AA$ within a few hundred nanoseconds or less. Such fast binding indicates that the clustering of positive charges in site 1 generates large electrostatic forces leading to longrange attraction of the negatively charged $\mathrm{N}$-terminal region of chemokine receptors. This, in turn, suggests that sulfotyrosine recognition through site 1 may be the first step in receptor recognition, followed by binding of the divergent regions/loops to the receptor's orthosteric pocket. 
Although our models offer a glimpse of how LukE might bind to ACKR1 and CCR5 orthosteric pocket, it should be emphasized that computational models are error-prone and thus might be inaccurate. Moreover, the timescale of the simulations used is limited and unable to account for potential large-scale receptor conformational changes that toxin binding may induce. For the same reason, it is not currently possible to determine from the data whether LukE- receptor binding might allosterically induce STEM release in LukE, which would be a necessary step to enable LukD binding and subsequent pore assembly without interprotomeric steric clashes (Yamashita, Kawai et al. 2011, Yamashita, Sugawara et al. 2014, Liu, Kozhaya et al. 2020). Thus, the proposed models might only represent initial encounter complexes that are subject to further conformational changes.

With these limitations in mind, a comparison of the proposed models of ACKR1-LukE and CCR5-LukE with the available (pre)pore structures of HIgAB, PVL, and lukGH in complex with human CD11b I-domain yields a few interesting observations:

Firstly, toxin-receptor and toxin-toxin interactions are mediated by distinct, non-overlapping surfaces, as was also observed for the lukGH - CD11b l-domain complex (Figure 8A and B). This indicates that the initial step of receptor recognition is compatible with subsequent toxin oligomerization.

Secondly, the depth of insertion of LukE into the receptors' orthosteric pocket is insufficient to allow the pore's $\beta$-barrel to fully span the membrane. Again, this might be due to model inaccuracies and/or conformational changes of the receptors unaccounted for in our modeling procedure. An alternative possibility is that receptors might serve as an assembly platform for the prepore structure but be released from the interaction prior to full pore formation. In support of this hypothesis, the crystal structure of PVL (pre)pore bound to fos-choline-14 has shown binding of 3 PC headgroups in different regions of LukF-PV corresponding to different membrane insertion depths, with the highest one compatible with the full membrane spanning pore (figure $\mathbf{8 C}$ ). When compared with the sulfotyrosine-bound crystal structures reported here, this would imply that site 2 is at membrane level, which would be incompatible with receptor binding. On the contrary, the 2 other PC binding sites, which have also been identified in lukD structures, are located in regions compatible with the level of membrane insertion implied by our ACKR1-LukE and CCR5-LukE models. Importantly, these observations are also consistent with the study by Haapasalo et al. which showed that C5a receptors dissociate from lukSF-PV upon pore formation, enabling renewed ligand binding and pore formation (Haapasalo, Wollman et al. 2019).

Finally, our ACKR1-LukE and CCR5-LukE models suggest that the bound toxin could make an acute angle with the membrane that may bring the preSTEM region closer to the lipid headgroups (figure $\mathbf{8 C}$ ), possibly playing some role in STEM release. 

made available under aCC-BY 4.0 International license.

\section{A}

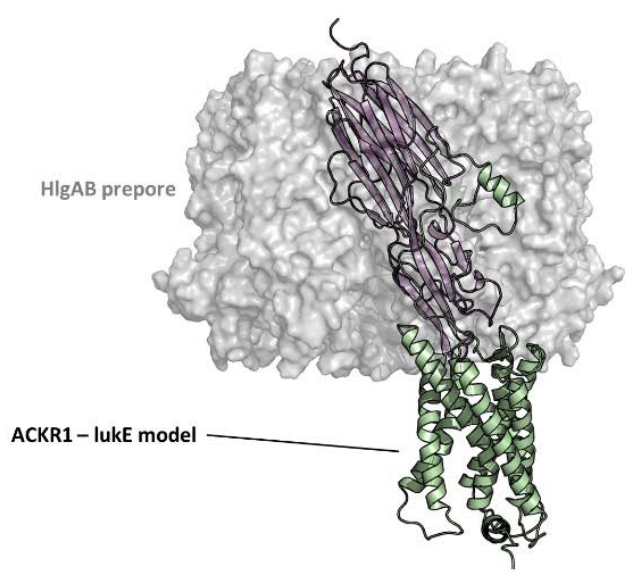

B

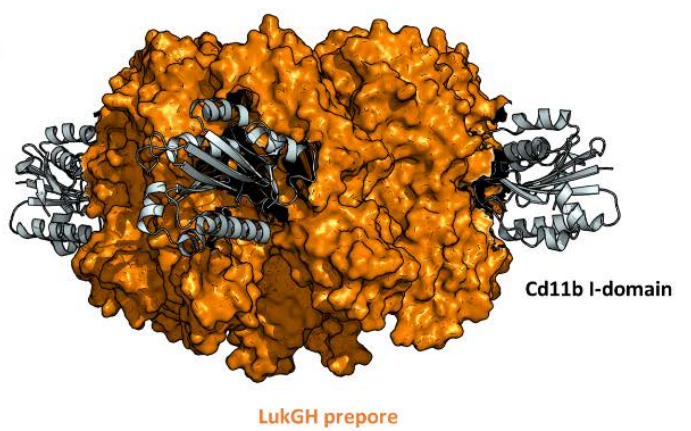

C

CCR2 sulfopeptide
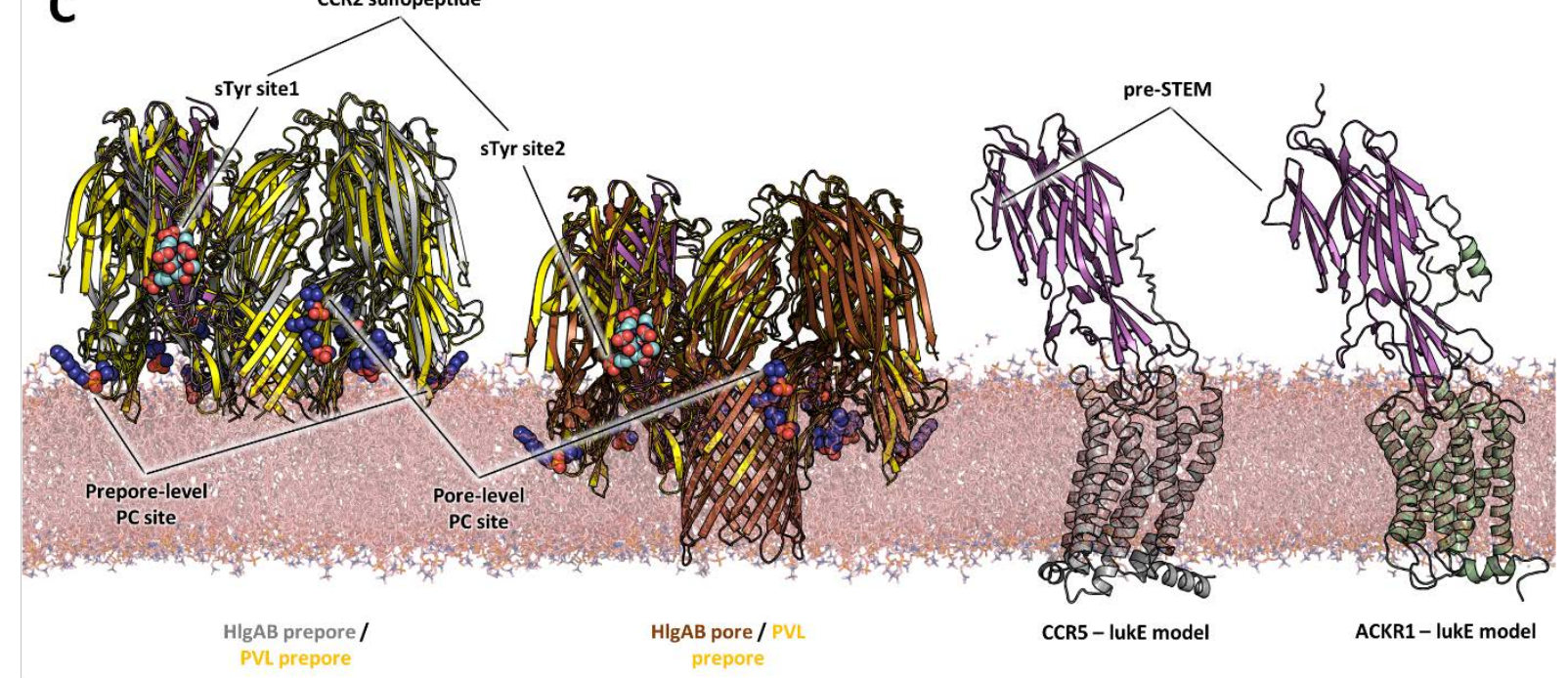

Figure 8: Comparisons of LukE-CCR2 peptide x-ray structure, ACKR1-LukE and CCR5-LukE models with existing $x$-ray structures. A. Structural alignment of LukE from the ACKR1-LukE model with the HIgAB prepore structure (PDB ID 4P1Y), illustrating that the $\mathrm{N}$-terminal region of ACKR1 binds to a surface located on the outer part of the prepore. The HIgAB prepore is shown as a semitransparent surface and the ACKR1-LukE model as cartoon. C. Xray structure of LukGH bound to human CD11b I-domain. The LukGH prepore is shown as a semitransparent surface and the CD11 b I-domain as cartoon. C. The HIgAB pore and prepore structures (PDB IDs 3B07 and 4P1Y) are shown in their membrane context using a POPC bilayer extracted from MDS simulations. On the left, the HIgAB pore and prepore structures are superimposed to the PVL prepore (PDB ID 6U3Y) bound with fos-choline14 (shown as blue spheres). One of the lukF-PV/HIgB protomer is omitted for clarity. The structural alignment highlights the presence of lipid headgroup binding sites on lukF-PV that correspond to different membrane insertion depths, which we interpreted as pore and prepore membrane levels. The LukE-CCR2 peptide structure is also superimposed onto the pore and prepore structures to indicate the location of the bound sulfotyrosines (shown as cyan spheres). In the case of a fully formed pore, sTyr sulfate at site 2 is located at the same level as the PC headgroups. On the right, the ACKR1-LukE and CCR5-LukE models are shown in their membrane context, and oriented to visualize the acute angle between LukE and the membrane. 
In summary, we have elucidated the structural basis for recognition of sulfotyrosine motifs located in the N-terminal region of leukotoxin receptors and used this data to propose models of the ACKR1-LukE and CCR5-LukE encounter complexes. Although the combination of crystallographic and mutagenesis data provide substantial validation for these models, they may still suffer from inaccuracies and could be further improved by additional interface mapping through biophysical approaches (such as HDX-MS or NMR for instance). They also constitute a starting point to design strategies to obtain high-resolution structural data, as, so far, the purified complexes were reluctant to LCP crystallography and cryoEM analyses. However, in the absence of high resolution cryoEM or x-ray structures of full-length GPCRtoxin complexes, these models help refine our understanding of the mechanisms driving receptor recognition and subsequent pore formation by SA leukotoxins. Sulfotyrosine site 1 additionally appears to have a unique architecture, which is distinct from the recognition motifs used by chemokines. The binding site is widely conserved across S-components, suggesting that this pocket might be a promising target for structure-based design of inhibitors preventing receptor recognition. Such inhibitors might be active against a broad range of SA strains expressing different leukotoxins.

\section{Materials and methods}

\section{Protein constructs and reagents}

The synthetic gene of Staphylococcus aureus LukEv (UniprotKB-Q2FXBO) residue 12-311 (the product of which was successfully expressed and crystallized in (Nocadello, Minasov et al. 2016)) was subcloned into popinE vector (OPPF-UK), resulting in a construct bearing a part of the $\mathrm{N}$-terminal signal sequence $\left({ }^{12}\right.$ SVGLIAPLA SPIQESRA $\left.{ }^{28}\right)$ and a C-terminal $\mathrm{K}(\mathrm{H})_{6}$-tag. For cell-based assays, we used SNAP-tagged ACKR1, CCR2 and CCR5 constructs. The synthetic genes of each receptor were subcloned into pcDNA3.1-SNAP vector in frame with the C-terminus of SNAP tag. d2-labelled CCL2 and CCL5 were purchased from Almac. P-cresol sulfate was purchased from Sigma-Aldrich (SMB00936).

\section{Expression and purification of LukE}

C-terminally (His) 6 -tagged LukE was expressed in BL21 (DE3) Escherichia coli cells (NEB). Transformed cells were grown at $37{ }^{\circ} \mathrm{C}$ in Terrific broth supplemented with $100 \mu \mathrm{g} / \mathrm{mL}$ ampicillin to a density of OD600 $=0.6$. Expression was then induced overnight at $18{ }^{\circ} \mathrm{C}$ by addition of $0.5 \mathrm{mM}$ IPTG. Cells were harvested by centrifugation $(3,000 \mathrm{rpm})$ and cell pellets were stored at $-80^{\circ} \mathrm{C}$ until purification. After thawing the frozen cell pellets, cells were lysed by sonication in a lysis buffer consisting of $10 \mathrm{mM}$ Tris $(\mathrm{pH} 8)$ and $150 \mathrm{mM} \mathrm{NaCl}$. Lysed cells were centrifuged $(16,000 \mathrm{rpm})$ and the supernatant was loaded onto a nickel NTA Agarose resin. The resin was washed with $20 \mathrm{CV}$ of wash buffer 1 consisting of 50 $\mathrm{mM}$ HEPES ( $\mathrm{pH} 7.5$ ) and $1 \mathrm{M} \mathrm{NaCl}$, and with $10 \mathrm{CV}$ of wash buffer 2 consisting of $50 \mathrm{mM}$ HEPES (pH 7.5), $150 \mathrm{mM} \mathrm{NaCl}$ and $40 \mathrm{mM}$ imidazole. Bound toxin was eluted with wash buffer 2 supplemented with $200 \mathrm{mM}$ imidazole. The eluted proteins were concentrated using $30 \mathrm{kDa}$ spin filters (Millipore) and further purified by size exclusion chromatography on a Superdex 200 Increase 10/300 column (GE Healthcare) in $50 \mathrm{mM}$ HEPES ( $\mathrm{pH} 7.5$ ) and $150 \mathrm{mM} \mathrm{NaCl}$. Monodisperse elution fractions were pooled and further concentrated prior to crystallization, SAXS and/or cell-based assays.

\section{Native mass spectrometry}

Prior to MS analysis, proteins were buffer exchanged into $200 \mathrm{mM}$ ammonium acetate buffer $\mathrm{pH} 7.4$ (Sigma) using Bio-Spin microcentrifuge columns (Bio-Rad Laboratories). Intact MS spectra were recorded on a Synapt G2-Si HDMS instrument (Waters Corporation) modified for high mass analysis 
and operated in ToF mode. Samples were introduced into the ion source using borosilicate emitters (Thermo Scientific). Optimized instrument parameters were as follows: capillary voltage $1.4 \mathrm{kV}$, sampling cone voltage $80 \mathrm{~V}$, offset voltage $80 \mathrm{~V}$, transfer collision voltage $25 \mathrm{~V}$ and argon flow rate 5 $\mathrm{mL} / \mathrm{min}$. Collision voltage in the trap was optimized between 50 and $110 \mathrm{~V}$. Data was processed using MassLynx v.4.2 (Waters). HEK293T cells (ATCC ${ }^{\circledR}$ CCRL-3216 ${ }^{\text {TM }}$, human embryonic kidney) were grown in Dulbecco's Modified Eagle Medium supplemented with $10 \% \mathrm{FBS}$ and $1 \%$ Penicillin/streptomycin at $37{ }^{\circ} \mathrm{C}$ with $5 \% \mathrm{CO}_{2}$. Transfections were performed using lipofectamine 2000 (Life technology) in polyornithine coated white 96-well, opaque-bottom plates. For CCR5 and ACKR1 constructs, cells were seeded into 6-well plates at a density of 300,000 cells/well before being transfected overnight with $100 \mathrm{ng} /$ well of SNAPCCR5 or SNAP-ACKR1. An empty PRK vector was added to obtain a total of 3ug of DNA/well. After 24 hours, cells were further seeded into 96 -well white, opaque plates at a density of 30,000 cells/well. CCR2 was transfected directly into 96 well plates by adding 2 ng of SNAP-CCR2 to 30,000 cells/well and empty PRK vector was added for a total $200 \mathrm{ng} /$ well of DNA. $24 \mathrm{~h}$ after seeding into 96-well plates, cells were washed with TagLite ${ }^{\circ}$ buffer (Cisbio, Codolet, France) and the receptors were labelled with SNAP-Lumi4-Tb (100 nM, Cisbio, Codolet, France) for $1 \mathrm{~h}$ at room temperature. Cells were washed four times with TagLite ${ }^{\circ}$ and then treated with increasing concentrations of LukE. $5 \mu \mathrm{M}$ of a fluorescent tracer was then added to wells and incubated for $4 \mathrm{hr}$ at $4^{\circ} \mathrm{C}$. CCL5-d2 was used as the tracer for CCR5 and ACKR1 and CCL2-d2 was used for CCR2. Fluorescence readouts were osberved on a Pherastar plate reader (BMG Labtech) or Spark $20 \mathrm{M}$ reader (Tecan): samples were illuminated at $337 \mathrm{~nm}$ and fluorescence was acquired at $620 \mathrm{~nm}$ (donor) and $665 \mathrm{~nm}$ (acceptor). The ratio of the signals (665/620) was calculated and plotted against toxin concentration. Dose-response curves were generated using GraphPad Prism $6^{\circ}$ (GraphPad Software, Inc., San Diego, CA).

\section{Small angle $x$-ray scattering}

Small angle X-ray scattering measurements of LukE at 1,2 and $4 \mathrm{mg} / \mathrm{ml}$ in $50 \mathrm{mM} \mathrm{HEPES} \mathrm{(pH} \mathrm{7.5)} \mathrm{and}$ $150 \mathrm{mM} \mathrm{NaCl}$ were performed at the BioSAXS beamline (BM29) of the European Synchrotron Radiation Facility (ESRF). Data was collected at $20^{\circ} \mathrm{C}$, a wavelength of $0.0995 \mathrm{~nm}$ and a sample-to-detector distance of $1 \mathrm{~m}$. The scattering from the buffer alone was measured before and after each sample measurement and was used for background subtraction with PRIMUS from the ATSAS package (Franke, Petoukhov et al. 2017).

Molecular dynamics and ensemble optimization of LUkE.

Starting coordinates for LukE were taken from the Apo2 structure. Missing terminal residues were added in extended conformations in Coot (Emsley and Cowtan 2004). MD simulations were performed using GROMACS 2020 (Hess, Kutzner et al. 2008) and either the AMBER99SB-ILDN* force field (Best and Hummer 2009, Lindorff-Larsen, Piana et al. 2010), or the AMBER99SBWS forcefields (Best, Zheng et al. 2014). At the beginning of each simulation, the protein was immersed in a box of SPC/E or TIP4P water, with a minimum distance of $1.0 \mathrm{~nm}$ between protein atoms and the edges of the box. 150 $\mathrm{mM} \mathrm{NaCl}$ were added using genion. Long range electrostatics were treated with the particle-mesh Ewald summation (Essmann, Perera et al. 1995). Bond lengths were constrained using the P-LINCS algorithm (Hess 2008). The integration time step was $5 \mathrm{fs}$. The v-rescale thermostat (Bussi, Donadio et al. 2007) and the Parrinello-Rahman barostat were used to maintain a temperature of $300 \mathrm{~K}$ and a pressure of $1 \mathrm{~atm}$. Each system was energy minimized using 1,000 steps of steepest descent and equilibrated for $200 \mathrm{ps}$ with restrained protein heavy atoms. A single $500 \mathrm{~ns}$ production simulation was run using each forcefield. RMSF and radius of gyration were calculated using GROMACS routines. Snapshots were extracted every 500 ps, resulting in a pool of 2,000 models. Theoretical SAXS patterns were calculated with the program CRYSOL (Svergun, Barberato et al. 1995) and ensemble fitting was performed with GAJOE (Bernado, Mylonas et al. 2007). 
Peptide synthesis

ACKR $1{ }^{34} D S F P D G D S Y G A N L E^{46}$ and CCR2 ${ }^{25}$ DsYDsYG ${ }^{29}$ sulfopeptides used in crystal soaking experiments were synthesized using solid-phase synthesis (SPPS) methodology with Fmoc/tBu protocol and all amino acids were incorporated into the sequence by the building block approach. Those synthesis was carried out by hand using the syringe technique on Fmoc-Rink Amide Resin (Amphisphere 40 RAM) with a substitution value of $0.35 \mathrm{mmol} / \mathrm{g}$. Side chain protecting groups that were used in this synthesis were $t \mathrm{Bu}$ for Asp,Glu and Ser; Trt for Asn and Fmoc-Tyr $\left(\mathrm{SO}_{3} \mathrm{Na}\right)-\mathrm{OH}$ was synthesized separately in solution. Fmoc groups for $\mathrm{N} \alpha$-protection were cleaved by treatment with $20 \%$ piperidine in DMF in two times. After each deprotection of Fmoc group or coupling step, resin was washed as follow: 3 times DMF, 2 times $\mathrm{MeOH}, 2$ times DMF and 2 times DCM. The appropriate amino acid (3 eq.) was dissolved in DMF in presence of HATU (2.9 eq.) and DIEA (4.5 eq.) for 5 minutes. This solution was then added to the resin and leaved under agitation at room temperature. Effectiveness of the reaction was monitored by disappearance of primary amino group at the $\mathrm{N}$ terminus of the growing peptide. This was checked by a negative Kaiser test or Chloranil test for proline residue. Durations of coupling steps ranged from 2 hours to overnight. After the last deprotection of Fmoc group, acetylation on $\mathrm{N}$-terminal group was performed twice for $15 \mathrm{~min}$ with a solution of DMF/Ac $2 \mathrm{O}(4: 1 ; \mathrm{v} / \mathrm{v})$ and $1 \%$ of DIEA.

Then after a wash step, the side-chain protection groups were removed and the peptides were cleaved from the resin using a mixture of $90 \%$ TFA and $10 \% \mathrm{H} 20$ for $2 \mathrm{~h}$ at $0^{\circ} \mathrm{C}\left({ }^{25} \mathrm{Ds} Y D s Y G^{29}\right)$ or $6 \mathrm{~h}$ at $0^{\circ} \mathrm{C}$ $\left({ }^{34}\right.$ DSFPDGDSYGANLE $\left.{ }^{46}\right)$. After filtration of the resin and concentration of filtrat at room temperature, crude peptide is precipitate by dropwise in diethylether at $0^{\circ} \mathrm{C}$. Crude peptide is then dissolve in a mix of water and acetonitrile. $\mathrm{pH}$ was adjusted above 9 with a solution of $\mathrm{NH}_{4} \mathrm{OH}$ aq $10 \%$ before to be lyophilisate.

Crude peptide was purified by HPLC in formiate buffer ( $\mathrm{pH} 4.5)$ and LC-MS analyses were performed by Electrospray negative mode.

\section{X-ray crystallography}

Crystallization was carried out by vapor diffusion using a Cartesian Technologies pipetting system (Walter, Diprose et al. 2005). LukE was concentrated to about $12 \mathrm{mg} / \mathrm{ml}(390 \mu \mathrm{M})$ in $25 \mathrm{mM} \mathrm{HEPES} \mathrm{pH}$ 7.5 and $75 \mathrm{mM} \mathrm{NaCl}$, and in the presence of 6 molar equivalents of the ACKR1 sulfopeptide. The protein crystallized after $3-7$ days at $20^{\circ} \mathrm{C}$ in $0.1 \mathrm{M}$ Imidazole. $\mathrm{HCl} \mathrm{pH} \mathrm{8.0,30 \%} \mathrm{(w/v)} \mathrm{MPD,} 10 \%(\mathrm{w} / \mathrm{v})$ PEG 4000 (apo1 condition), or in $0.1 \mathrm{M}$ Ammonium sulfate, $0.05 \mathrm{M}$ Magnesium sulfate heptahydrate, 0.1 M Sodium citrate pH 5.5 and 22.5 \% v/v PEG Smear Medium (BCS screen from Molecular Dimensions) (apo2 condition). These crystals led to the apo structures (apo1 and 2, respectively). No sign of the sulfopeptide was observed in the electron density. Consequently, new crystals were produced in the apo1 crystallization condition in order to perform crystal soaking experiments. A single crystal was obtained and broken into 3 pieces, each of which were incubated in mother liquor supplemented with either $p$-cresol sulfate, CCR2 or ACKR1 sulfopeptide (all in powder form) for 3 days. Crystals were then frozen in liquid nitrogen after being soaked in mineral oil. Diffraction data were recorded on the MASSIF-1 beamline at the ESRF, Grenoble, France (soaks), or on the X06DA beamline at the Swiss Light Source (apo $1 \& 2$ ). All data were automatically processed by xia2 (Winter, Lobley et al. 2013), and scaled intensities from XDS (Kabsch 2010) were merged using AIMLESS (Evans and Murshudov 2013) using appropriate high resolution cut-offs to yield the final datasets.

Structural determination was initiated by molecular replacement using the previously published LukE structure (PDB ID: 3ROH) as a search model in PHASER (McCoy, Grosse-Kunstleve et al. 2007). The solution was subjected to repetitive rounds of restrained refinement in PHENIX (Adams, Afonine et al. 2010) and Autobuster (Blanc, Roversi et al. 2004) and manual building in COOT (Emsley, Lohkamp et al. 2010). TLS parameters were included in the final round of refinement. The CCP4 program suite (Winn, Ballard et al. 2011) was used for coordinate manipulations. The structures were validated with Molprobity (Chen, Arendall et al. 2010). Refinement statistics are given in Table 1, and final refined coordinates and structure factors have been deposited in the PDB with accession codes 7P8T (apo 1), 
7P8S (apo 2), 7P8U (p-cresol sulfate soak), 7P8X (CCR2 ${ }^{25}$ DsYDsYG $^{29}$ soak), and 7P93 (ACKR1 ${ }^{34}$ DSFPDGDSYGANLE ${ }^{46}$ soak).

\section{Protein-protein docking}

Computational docking of LukE onto ACKR1 and CCR5 was performed using the information-driven docking software HADDOCK 2.4, which is available as a webserver (van Zundert, Rodrigues et al. 2016). In the absence of a high-resolution structure for ACKR1, we used an ensemble of 6 MD simulationderived models that have been partially validated using H/DX mass spectrometry data in our previous ACKR1 study (ref biorxiv). Each model was docked to the LukE apo1 structure using default parameters ( 6 runs). We used as active residues Cys51, Glu202 and Arg267 of ACKR1, based on the work by Spaan and colleagues (Spaan, Reyes-Robles et al. 2015). The active residues of LukE were defined as Tyr96, Asp197, Pro215 and Arg275, which are located respectively in loop 1, 2, 3 and 4. Each of these loops contain residues that are divergent between different leukotoxins and that have been implicated in LukED hemolysis in (Vasquez, Lubkin et al. 2020) and/ or in (Peng, Takeshita et al. 2018). In the case of CCR5 for which several experimental structures are available, the CCR5 chain was extracted from 5 UIW (CCR5-CCL5 complex) or 6MEO (CCR5- gp120-CD4 complex). Each CCR5 chain was docked to the LukE apo1 or apo2 structure with HADDOCK (4 runs). The CCR5 active residues were Lys171, Glu172, Ser179, Arg274 and Asp276, which were found to mediate LukED toxicity in CCR5 ${ }^{+}$cells by Tam et al. (Tam, Schultz et al. 2016). Tyr96 and Arg275 of LukE were selected as active residues based on (Reyes-Robles, Alonzo et al. 2013, Tam, Schultz et al. 2016). omitted from the models in order to avoid potential steric clashes with the toxin. The best scoring model from the top HADDOCK cluster of the best scoring run was then selected for molecular dynamics refinement.

\section{Classical molecular dynamics simulations}

Prior to MD simulations, the N-terminal region of the receptors (residues 8-50 of ACKR1 and residues 1-20 of CCR5) were added to the selected models, with ACKR1 Tyr41, and CCR5 Tyr3, 10, 14 and 15 in the sulfated form. The ACKR1-LukE and CCR5-LukE MD systems were then set up using the CHARMMGUI membrane builder ( $\mathrm{Wu}$, Cheng et al. 2014). The starting models were each inserted into a hydrated, equilibrated bilayer composed of approximately 500 2-Oleoyl-1-palmitoyl-sn-glycero-3phosphocholine (POPC) and 100 cholesterol molecules. Sodium and chloride ions were added to neutralize the system, reaching a final concentration of approximately $150 \mathrm{mM}$. Molecular dynamics calculations were performed in GROMACS 2020 using the CHARMM36m force field (Huang, Rauscher et al. 2017) and the CHARMM TIP3P water model. Forcefield parameters for sulfotyrosine were obtained by combining CHARMM topologies from related molecules. The input systems were subjected to energy minimization, equilibration and production simulation using the GROMACS input scripts generated by CHARMM-GUI (Lee, Cheng et al. 2016). The temperature and pressure were held at $310.15 \mathrm{~K}$ and $1 \mathrm{bar}$, respectively. During production simulations an NPT ensemble was used with semi-isotropic pressure coupling via the Parrinello-Rahman barostat method while the Nose-Hoover thermostat was used to maintain a temperature of $310.15 \mathrm{~K}$. A leapfrog integration scheme was used, and all bonds were constrained allowing for a time-step of 2 ps to be used during NPT equilibration and production MD simulations. For each system, we performed 3 production runs and subsequently analyzed the resulting trajectories using GROMACS tools to yield interface root mean square deviations (iRMSD.), complex buried surface area (BSA), number of interchain hydrogen bonds and inter-residue distances.

\section{AWH molecular dynamics simulations}

The accelerated weight histogram (AWH) method (Lindahl, Lidmar et al. 2014) is an adaptive biasing method implemented in GROMACS (Hess, Kutzner et al. 2008). This method enables the calculation of potential of mean force (PMF) profiles along (a) chosen reaction coordinate(s), and functions by applying a time-dependent biasing potential that flattens free energy barriers along the reaction path. 
In the case of ACKR1-LukE, the sTyr 41 - Arg263 and sTyr41 - Tyr269 COM distances were defined as a two-dimensional reaction coordinate to report on site 1 and site 2 sulfotyrosine interactions. The sampling interval was $0.2-3.5 \mathrm{~nm}$. The force constant and initial error for AWH calculations were set to $10000 \mathrm{~kJ} / \mathrm{mol} / \mathrm{nm}^{2}$ and $10 \mathrm{~kJ} / \mathrm{mol}$, respectively. An estimated diffusion parameter of $1.10^{-5} \mathrm{~nm}^{2} / \mathrm{ps}$ was used for each coordinate dimension. A free energy cutoff of $50 \mathrm{~kJ} / \mathrm{mol}$ was applied to the AWH target distribution to avoid sampling of very high free energy regions. A single trajectory of $2.147 \mu \mathrm{s}$ was calculated in GROMACS. The free energy profiles at different simulation times were constructed using the gmx awh program included in GROMACS. The final PMF seemed converged with no major change taking place after about $1.8 \mu \mathrm{s}$ when it exited the initial stage. The estimated AWH target error at the end of the simulation was $\sim 2.2 \mathrm{~kJ} / \mathrm{mol}$ (down from $10 \mathrm{~kJ} / \mathrm{mol}$ at the start).

\section{Acknowledgements}

This work was supported by the french Agence Nationale de la Recherche (project ANR-17-CE15-000201, CHEMSPEC). The authors would like to thank the staff of beamline BM29 and MASSIF-1 at the European Synchrotron Radiation Facility (Grenoble, France) for assistance with X-ray data collection. The authors would also like to thank the staff of beamline X06DA at the PSI for assistance with crystal testing and data collection. The FP7 WeNMR (project\# 261572), H2020 West-Life (project\# 675858), the EOSC-hub (project\# 777536) and the EGI-ACE (project\# 101017567) European e-Infrastructure projects are acknowledged for the use of their web portals, which make use of the EGI infrastructure with the dedicated support of CESNET-MCC, INFN-PADOVA-STACK, INFN-LNL-2, NCG-INGRID-PT, TWNCHC, CESGA, IFCA-LCG2, UA-BITP, SURFsara and NIKHEF, and the additional support of the national GRID Initiatives of Belgium, France, Italy, Germany, the Netherlands, Poland, Portugal, Spain, UK, Taiwan and the US Open Science Grid. Peptide synthesis was performed using Synbio3 platform supported by GIS IBISA and ITMO Cancer (Montpellier, France). We additionally would like to thank Marc Leyrat for his help in making movie S1.

\section{Competing interests}

The authors declare that no competing interests exist.

\section{References}

Adams, P. D., P. V. Afonine, G. Bunkoczi, V. B. Chen, I. W. Davis, N. Echols, J. J. Headd, L. W. Hung, G. J. Kapral, R. W. Grosse-Kunstleve, A. J. McCoy, N. W. Moriarty, R. Oeffner, R. J. Read, D. C. Richardson, J. S. Richardson, T. C. Terwilliger and P. H. Zwart (2010). "PHENIX: a comprehensive Python-based system for macromolecular structure solution." Acta Crystallogr D Biol Crystallogr 66(Pt 2): 213-221.

Alonzo, F., 3rd, L. Kozhaya, S. A. Rawlings, T. Reyes-Robles, A. L. DuMont, D. G. Myszka, N. R. Landau, D. Unutmaz and V. J. Torres (2013). "CCR5 is a receptor for Staphylococcus aureus leukotoxin ED." Nature 493(7430): 51-55.

Badarau, A., H. Rouha, S. Malafa, M. B. Battles, L. Walker, N. Nielson, I. Dolezilkova, A. Teubenbacher, S. Banerjee, B. Maierhofer, S. Weber, L. Stulik, D. T. Logan, M. Welin, I. Mirkina, C. Pleban, G. Zauner, K. Gross, M. Jagerhofer, Z. Magyarics and E. Nagy (2016). "Context matters: The importance of dimerization-induced conformation of the LukGH leukocidin of Staphylococcus aureus for the generation of neutralizing antibodies." MAbs 8(7): 1347-1360.

Bernado, P., E. Mylonas, M. V. Petoukhov, M. Blackledge and D. I. Svergun (2007). "Structural characterization of flexible proteins using small-angle X-ray scattering." J Am Chem Soc 129(17): 5656-5664. 
Bernardi, R. C., M. C. R. Melo and K. Schulten (2015). "Enhanced sampling techniques in molecular dynamics simulations of biological systems." Biochimica et Biophysica Acta (BBA) - General Subjects 1850(5): 872-877.

Best, R. B. and G. Hummer (2009). "Optimized Molecular Dynamics Force Fields Applied to the Helixâ^’Coil Transition of Polypeptides." The Journal of Physical Chemistry B 113(26): 9004-9015. Best, R. B., W. Zheng and J. Mittal (2014). "Balanced Protein-Water Interactions Improve Properties of Disordered Proteins and Non-Specific Protein Association." J Chem Theory Comput 10(11): 51135124.

Blanc, E., P. Roversi, C. Vonrhein, C. Flensburg, S. M. Lea and G. Bricogne (2004). "Refinement of severely incomplete structures with maximum likelihood in BUSTER-TNT." Acta Crystallogr D Biol Crystallogr 60(Pt 12 Pt 1): 2210-2221.

Bussi, G., D. Donadio and M. Parrinello (2007). "Canonical sampling through velocity rescaling " J. Chem. Phys. 126: 014101.

Chen, V. B., W. B. Arendall, 3rd, J. J. Headd, D. A. Keedy, R. M. Immormino, G. J. Kapral, L. W. Murray, J. S. Richardson and D. C. Richardson (2010). "MolProbity: all-atom structure validation for macromolecular crystallography." Acta Crystallogr D Biol Crystallogr 66(Pt 1): $12-21$.

DeLeo, F. R., B. A. Diep and M. Otto (2009). "Host defense and pathogenesis in Staphylococcus aureus infections." Infect Dis Clin North Am 23(1): 17-34.

DuMont, A. L., P. Yoong, C. J. Day, F. Alonzo, 3rd, W. H. McDonald, M. P. Jennings and V. J. Torres (2013). "Staphylococcus aureus LukAB cytotoxin kills human neutrophils by targeting the CD11b subunit of the integrin Mac-1." Proc Natl Acad Sci U S A 110(26): 10794-10799.

Emsley, P. and K. Cowtan (2004). "Coot: model-building tools for molecular graphics." Acta crystallographica. Section D, Biological crystallography 60(Pt 12 Pt 1): 2126-2132.

Emsley, P., B. Lohkamp, W. G. Scott and K. Cowtan (2010). "Features and development of Coot." Acta Crystallogr D Biol Crystallogr 66(Pt 4): 486-501.

Essmann, U., L. Perera, M. L. Berkowitz, T. Darden, H. Lee and L. G. Pedersen (1995). "A smooth particle mesh Ewald method." Journal of Chemical Physics 103(19): 8577-8593.

Evans, P. R. and G. N. Murshudov (2013). "How good are my data and what is the resolution?" Acta Crystallogr D Biol Crystallogr 69(Pt 7): 1204-1214.

Farzan, M., T. Mirzabekov, P. Kolchinsky, R. Wyatt, M. Cayabyab, N. P. Gerard, C. Gerard, J. Sodroski and H. Choe (1999). "Tyrosine sulfation of the amino terminus of CCR5 facilitates HIV-1 entry." Cell 96(5): 667-676.

Franke, D., M. V. Petoukhov, P. V. Konarev, A. Panjkovich, A. Tuukkanen, H. D. T. Mertens, A. G. Kikhney, N. R. Hajizadeh, J. M. Franklin, C. M. Jeffries and D. I. Svergun (2017). "ATSAS 2.8: a comprehensive data analysis suite for small-angle scattering from macromolecular solutions." J Appl Crystallogr 50(Pt 4): 1212-1225.

Grison, C. M., C. Leyrat, P. Lambey, S. Jeannot, E. Del Nero, S. Fontanel, F. Peysson, J. Heuninck, R. Sounier, T. Durroux, S. Granier and C. Bechara (2021). "Mechanisms of GPCR hijacking by \&lt;em\&gt;Staphylococcus aureus\&lt;/em\&gt." bioRxiv: 2021.2002.2008.430206.

Gryp, T., R. Vanholder, M. Vaneechoutte and G. Glorieux (2017). "p-Cresyl Sulfate." Toxins (Basel) 9(2).

Guillet, V., P. Roblin, S. Werner, M. Coraiola, G. Menestrina, H. Monteil, G. Prevost and L. Mourey (2004). "Crystal structure of leucotoxin S component: new insight into the Staphylococcal beta-barrel pore-forming toxins." J Biol Chem 279(39): 41028-41037.

Haapasalo, K., A. J. M. Wollman, C. J. C. de Haas, K. P. M. van Kessel, J. A. G. van Strijp and M. C. Leake (2019). "Staphylococcus aureus toxin LukSF dissociates from its membrane receptor target to enable renewed ligand sequestration." FASEB J 33(3): 3807-3824.

Hess, B. (2008). "P-lincs: A parallel linear constraint solver for molecular simulation." J. Chem. Theory Comput 4(1): 116-122.

Hess, B., C. Kutzner, D. van der Spoel and E. Lindahl (2008). "GROMACS 4: Algorithms for Highly Efficient, Load-Balanced, and Scalable Molecular Simulation." J Chem Theory Comput 4(3): 435-447. 
Hess, B., C. Kutzner, D. van der Spoel and E. Lindahl (2008). "GROMACS 4: Algorithms for Highly Efficient, Load-Balanced, and Scalable Molecular Simulation." Journal of Chemical Theory and Computation 4(3): 435-447.

Huang, J., S. Rauscher, G. Nawrocki, T. Ran, M. Feig, B. L. de Groot, H. Grubmuller and A. D. MacKerell, Jr. (2017). "CHARMM36m: an improved force field for folded and intrinsically disordered proteins." Nat Methods 14(1): 71-73.

Jandova, Z., A. V. Vargiu and A. M. J. J. Bonvin (2021). "Native or non-native protein-protein docking models? Molecular dynamics to the rescue." bioRxiv: 2021.2004.2002.438171.

Jen, C. H., K. L. Moore and J. A. Leary (2009). "Pattern and temporal sequence of sulfation of CCR5 Nterminal peptides by tyrosylprotein sulfotransferase-2: an assessment of the effects of $\mathrm{N}$-terminal residues." Biochemistry 48(23): 5332-5338.

Kabsch, W. (2010). "Xds." Acta Crystallogr D Biol Crystallogr 66(Pt 2): 125-132.

Kong, C., H. M. Neoh and S. Nathan (2016). "Targeting Staphylococcus aureus Toxins: A Potential form of Anti-Virulence Therapy." Toxins (Basel) 8(3).

Laventie, B. J., F. Guerin, L. Mourey, M. Y. Tawk, E. Jover, L. Maveyraud and G. Prevost (2014).

"Residues essential for Panton-Valentine leukocidin S component binding to its cell receptor suggest both plasticity and adaptability in its interaction surface." PLoS One 9(3): e92094. Lee, J., X. Cheng, J. M. Swails, M. S. Yeom, P. K. Eastman, J. A. Lemkul, S. Wei, J. Buckner, J. C. Jeong, Y. Qi, S. Jo, V. S. Pande, D. A. Case, C. L. Brooks, 3rd, A. D. MacKerell, Jr., J. B. Klauda and W. Im (2016). "CHARMM-GUI Input Generator for NAMD, GROMACS, AMBER, OpenMM, and CHARMM/OpenMM Simulations Using the CHARMM36 Additive Force Field." J Chem Theory Comput 12(1): 405-413.

Lindahl, V., J. Lidmar and B. Hess (2014). "Accelerated weight histogram method for exploring free energy landscapes." The Journal of Chemical Physics 141(4): 044110.

Lindorff-Larsen, K., S. Piana, K. Palmo, P. Maragakis, J. L. Klepeis, R. O. Dror and D. E. Shaw (2010). "Improved side-chain torsion potentials for the Amber ff99SB protein force field." Proteins 78(8): 1950-1958.

Liu, J., L. Kozhaya, V. J. Torres, D. Unutmaz and M. Lu (2020). "Structure-based discovery of a smallmolecule inhibitor of methicillin-resistant Staphylococcus aureus virulence." J Biol Chem 295(18): 5944-5959.

McCoy, A. J., R. W. Grosse-Kunstleve, P. D. Adams, M. D. Winn, L. C. Storoni and R. J. Read (2007). "Phaser crystallographic software." J Appl Crystallogr 40(Pt 4): 658-674.

Nariya, H. and Y. Kamio (1997). "Identification of the minimum segment essential for the H gamma IIspecific function of staphylococcal gamma-hemolysin." Biosci Biotechnol Biochem 61(10): 1786-1788. Nocadello, S., G. Minasov, L. Shuvalova, I. Dubrovska, E. Sabini, F. Bagnoli, G. Grandi and W. F. Anderson (2016). "Crystal structures of the components of the Staphylococcus aureus leukotoxin ED." Acta Crystallogr D Struct Biol 72(Pt 1): 113-120.

Oliveira, D., A. Borges and M. Simoes (2018). "Staphylococcus aureus Toxins and Their Molecular Activity in Infectious Diseases." Toxins (Basel) 10(6).

Olson, R., H. Nariya, K. Yokota, Y. Kamio and E. Gouaux (1999). "Crystal structure of staphylococcal LukF delineates conformational changes accompanying formation of a transmembrane channel." $\underline{\text { Nat }}$ Struct Biol 6(2): 134-140.

Pedelacq, J. D., L. Maveyraud, G. Prevost, L. Baba-Moussa, A. Gonzalez, E. Courcelle, W. Shepard, H. Monteil, J. P. Samama and L. Mourey (1999). "The structure of a Staphylococcus aureus leucocidin component (LukF-PV) reveals the fold of the water-soluble species of a family of transmembrane pore-forming toxins." Structure 7(3): 277-287.

Peng, Z., M. Takeshita, N. Shibata, H. Tada, Y. Tanaka and J. Kaneko (2018). "Rim domain loops of staphylococcal beta-pore forming bi-component toxin S-components recognize target human erythrocytes in a coordinated manner." J Biochem 164(2): 93-102.

Preobrazhensky, A. A., S. Dragan, T. Kawano, M. A. Gavrilin, I. V. Gulina, L. Chakravarty and P. E. Kolattukudy (2000). "Monocyte chemotactic protein-1 receptor CCR2B is a glycoprotein that has tyrosine sulfation in a conserved extracellular N-terminal region." J Immunol 165(9): 5295-5303. 
Reyes-Robles, T., F. Alonzo, 3rd, L. Kozhaya, D. B. Lacy, D. Unutmaz and V. J. Torres (2013). "Staphylococcus aureus leukotoxin ED targets the chemokine receptors CXCR1 and CXCR2 to kill leukocytes and promote infection." Cell Host Microbe 14(4): 453-459.

Roblin, P., V. Guillet, O. Joubert, D. Keller, M. Erard, L. Maveyraud, G. Prevost and L. Mourey (2008). "A covalent S-F heterodimer of leucotoxin reveals molecular plasticity of beta-barrel pore-forming toxins." Proteins 71(1): 485-496.

Spaan, A. N., T. Henry, W. J. M. van Rooijen, M. Perret, C. Badiou, P. C. Aerts, J. Kemmink, C. J. C. de Haas, K. P. M. van Kessel, F. Vandenesch, G. Lina and J. A. G. van Strijp (2013). "The staphylococcal toxin Panton-Valentine Leukocidin targets human C5a receptors." Cell Host Microbe 13(5): 584-594. Spaan, A. N., T. Reyes-Robles, C. Badiou, S. Cochet, K. M. Boguslawski, P. Yoong, C. J. Day, C. J. de Haas, K. P. van Kessel, F. Vandenesch, M. P. Jennings, C. Le Van Kim, Y. Colin, J. A. van Strijp, T. Henry and V. J. Torres (2015). "Staphylococcus aureus Targets the Duffy Antigen Receptor for Chemokines (DARC) to Lyse Erythrocytes." Cell Host Microbe 18(3): 363-370.

Spaan, A. N., J. A. G. van Strijp and V. J. Torres (2017). "Leukocidins: staphylococcal bi-component pore-forming toxins find their receptors." Nat Rev Microbiol 15(7): 435-447.

Spaan, A. N., M. Vrieling, P. Wallet, C. Badiou, T. Reyes-Robles, E. A. Ohneck, Y. Benito, C. J. de Haas, C. J. Day, M. P. Jennings, G. Lina, F. Vandenesch, K. P. van Kessel, V. J. Torres, J. A. van Strijp and T. Henry (2014). "The staphylococcal toxins gamma-haemolysin AB and CB differentially target phagocytes by employing specific chemokine receptors." Nat Commun 5: 5438.

Svergun, D., C. Barberato and M. H. J. Koch (1995). "CRYSOL-a program to evaluate X-ray solution scattering of biological macromolecules from atomic coordinates." Journal of Applied Crystallography 28(6): 768-773.

Tam, K., M. Schultz, T. Reyes-Robles, B. Vanwalscappel, J. Horton, F. Alonzo, 3rd, B. Wu, N. R. Landau and V. J. Torres (2016). "Staphylococcus aureus Leukocidin LukED and HIV-1 gp120 Target Different Sequence Determinants on CCR5." $\underline{\mathrm{mBio}}$ 7(6).

Tan, J. H. Y., J. P. Ludeman, J. Wedderburn, M. Canals, P. Hall, S. J. Butler, D. Taleski, A. Christopoulos, M. J. Hickey, R. J. Payne and M. J. Stone (2013). "Tyrosine sulfation of chemokine receptor CCR2 enhances interactions with both monomeric and dimeric forms of the chemokine monocyte chemoattractant protein-1 (MCP-1)." J Biol Chem 288(14): 10024-10034.

Tong, S. Y., J. S. Davis, E. Eichenberger, T. L. Holland and V. G. Fowler, Jr. (2015). "Staphylococcus aureus infections: epidemiology, pathophysiology, clinical manifestations, and management." Clin Microbiol Rev 28(3): 603-661.

Tromp, A. T., M. Van Gent, P. Abrial, A. Martin, J. P. Jansen, C. J. C. De Haas, K. P. M. Van Kessel, B. W. Bardoel, E. Kruse, E. Bourdonnay, M. Boettcher, M. T. McManus, C. J. Day, M. P. Jennings, G. Lina, F. Vandenesch, J. A. G. Van Strijp, R. J. Lebbink, P. A. Haas, T. Henry and A. N. Spaan (2018). "Human CD45 is an F-component-specific receptor for the staphylococcal toxin Panton-Valentine leukocidin." Nat Microbiol 3(6): 708-717.

Tromp, A. T., M. Van Gent, J. P. Jansen, L. M. Scheepmaker, A. Velthuizen, C. J. C. De Haas, K. P. M. Van Kessel, B. W. Bardoel, M. Boettcher, M. T. McManus, J. A. G. Van Strijp, R. J. Lebbink, P. A. Haas and A. N. Spaan (2020). "Host-Receptor Post-Translational Modifications Refine Staphylococcal Leukocidin Cytotoxicity." Toxins (Basel) 12(2).

Trstenjak, N., D. Milic, M. A. Graewert, H. Rouha, D. Svergun, K. Djinovic-Carugo, E. Nagy and A. Badarau (2020). "Molecular mechanism of leukocidin GH-integrin CD11b/CD18 recognition and species specificity." Proc Natl Acad Sci U S A 117(1): 317-327.

van Zundert, G. C. P., J. Rodrigues, M. Trellet, C. Schmitz, P. L. Kastritis, E. Karaca, A. S. J. Melquiond, M. van Dijk, S. J. de Vries and A. Bonvin (2016). "The HADDOCK2.2 Web Server: User-Friendly Integrative Modeling of Biomolecular Complexes." J Mol Biol 428(4): 720-725.

Vasquez, M. T., A. Lubkin, T. Reyes-Robles, C. J. Day, K. A. Lacey, M. P. Jennings and V. J. Torres (2020). "Identification of a domain critical for Staphylococcus aureus LukED receptor targeting and lysis of erythrocytes." J Biol Chem 295(50): 17241-17250.

Walter, T. S., J. M. Diprose, C. J. Mayo, C. Siebold, M. G. Pickford, L. Carter, G. C. Sutton, N. S. Berrow, J. Brown, I. M. Berry, G. B. Stewart-Jones, J. M. Grimes, D. K. Stammers, R. M. Esnouf, E. Y. Jones, R. J. 
Owens, D. I. Stuart and K. Harlos (2005). "A procedure for setting up high-throughput nanolitre crystallization experiments. Crystallization workflow for initial screening, automated storage, imaging and optimization." Acta Crystallogr D Biol Crystallogr 61(Pt 6): 651-657. Winn, M. D., C. C. Ballard, K. D. Cowtan, E. J. Dodson, P. Emsley, P. R. Evans, R. M. Keegan, E. B. Krissinel, A. G. Leslie, A. McCoy, S. J. McNicholas, G. N. Murshudov, N. S. Pannu, E. A. Potterton, H. R. Powell, R. J. Read, A. Vagin and K. S. Wilson (2011). "Overview of the CCP4 suite and current developments." Acta Crystallogr D Biol Crystallogr 67(Pt 4): 235-242. Winter, G., C. M. Lobley and S. M. Prince (2013). "Decision making in xia2." Acta Crystallogr D Biol Crystallogr 69(Pt 7): 1260-1273. Wu, E. L., X. Cheng, S. Jo, H. Rui, K. C. Song, E. M. Davila-Contreras, Y. Qi, J. Lee, V. Monje-Galvan, R. M. Venable, J. B. Klauda and W. Im (2014). "CHARMM-GUI Membrane Builder toward realistic biological membrane simulations." J Comput Chem 35(27): 1997-2004. Yamashita, D., T. Sugawara, M. Takeshita, J. Kaneko, Y. Kamio, I. Tanaka, Y. Tanaka and M. Yao (2014). "Molecular basis of transmembrane beta-barrel formation of staphylococcal pore-forming toxins." Nat Commun 5: 4897.

Yamashita, K., Y. Kawai, Y. Tanaka, N. Hirano, J. Kaneko, N. Tomita, M. Ohta, Y. Kamio, M. Yao and I. Tanaka (2011). "Crystal structure of the octameric pore of staphylococcal gamma-hemolysin reveals the beta-barrel pore formation mechanism by two components." Proc Natl Acad Sci U S A 108(42): 17314-17319.

Zwier, J. M., T. Roux, M. Cottet, T. Durroux, S. Douzon, S. Bdioui, N. Gregor, E. Bourrier, N. Oueslati, L. Nicolas, N. Tinel, C. Boisseau, P. Yverneau, F. Charrier-Savournin, M. Fink and E. Trinquet (2010). "A fluorescent ligand-binding alternative using Tag-lite(R) technology." J Biomol Screen 15(10): 1248- 